\title{
Scyliorhinus hachijoensis, a new species of catshark from the Izu Islands, Japan (Carcharhiniformes: Scyliorhinidae)
}

\author{
NANAMI ITO ${ }^{1 *}$, MIHO FUJII ${ }^{2}$, KENJI NOHARA ${ }^{1,3} \&$ SHO TANAKA $^{1,3}$ \\ ${ }^{1}$ Graduate School of Oceanography, Tokai University, 3-20-1, Orido, Shimizu-ku, Shizuoka-shi, Shizuoka, Japan, 424-8610. \\ ${ }^{2}$ Shimoda Floating Aquarium,3-22-31, Shimoda-shi, Shizuoka, Japan, 415-0023. \\ Miho Fujii: |"| moramora1010@outlook.jp; ○ https://orcid.org/0000-0002-0785-2578 \\ ${ }^{3}$ School of Marine Science and Technology, Tokai University, 3-20-1, Orido, Shimizu-ku, Shizuoka-shi, Shizuoka, Japan, 424-8610. \\ Kenji Nohara: "knohara@tokai-u.jp; @ https://orcid.org/0000-0001-9279-3797 \\ Sho Tanaka: "="sho@scc.u-tokai.ac.jp; @ https://orcid.org/0000-0003-3707-3417 \\ *Corresponding author. ”itonnm78@gmail.com; ㄴttps://orcid.org/0000-0001-7935-8029
}

\begin{abstract}
A new species of catshark genus Scyliorhinus, S. hachijoensis sp. nov., is described for the islands of Mikurajima, Hachijojima, and Torishima in southeastern Japan. Scyliorhinus hachijoensis has clasper hooks, which is a common feature in males of the most closely related species (S. torazame), but is distinguished by its coloration (presence of dark spots), the height of its anal fin (higher than the caudal peduncle), and the shape of pectoral and pelvic fins, and dermal denticles. Molecular data also corroborates the new species as a distinct and monophyletic taxon by nucleotide sequence analysis of three mitochondrial DNA regions.
\end{abstract}

Key words: Scyliorhinus torazame, Scyliorhinus hachijoensis sp. nov., Chondrichthyes, morphology

\section{Introduction}

The catshark genus Scyliorhinus Blainville, 1816 consists of 16 benthic shark species with characteristic coloration such as light spots, dark spots and dark saddles, and are distributed in temperate to tropical waters around the world. These species inhabit depths ranging from the coast to the upper continental slope, and most species live at depths of less than 500 m (Springer 1979; Ebert et al. 2013a; Weigmann 2016; Soares \& de Carvalho 2019). The main morphological characters of Scyliorhinus are the presence of a laterally projected flap on the upper lip margin that covers the lower labial furrow, the presence of a pelvic apron in males, and an extension of the pelvic apron that is more than half the length of the pelvic inner margin (Soares \& de Carvalho 2019, 2020).

The catshark species S. torazame (Tanaka, 1908) and S. tokubee Shirai, Hagiwara \& Nakaya, 1992 have been reported from Japanese coastal waters, but $S$. tokubee was considered a junior synonym of $S$. torazame by Soares $\&$ de Carvalho (2019). Scyliorhinus torazame is distinguished from other Scyliorhinus species by the presence of clasper hooks in males (Soares \& de Carvalho 2019). Scyliorhinus torazame inhabits continental shelves (0-300 m depth), and has been found across Japan from Hokkaido to Okinawa, also occuring in South Korea, the East China Sea, and Taiwan (Nakaya 1975; Springer 1979; Compagno 1984; Shirai et al. 1992; Kim et al. 2009; Ebert et al. 2013a, b; Dyldin 2015; Soares \& de Carvalho 2019).

Specimens that appear to be similar to $S$. torazame have been captured off the islands of Torishima and Mikurajima (Hagiwara 1993). These specimens were considered to belong to a different species because they were captured from deep water (ca. 450-500 m depth) and differed in color pattern and in the shape of their egg cases. These specimens have been kept in the Shimoda Floating Aquarium, Shizuoka, since they were first discovered. However, their precise identification has not been investigated for about three decades. Here, we assess the morphological and genetic characters of Scyliorhinus sp. specimens captured from the islands of Mikurajima, Hachijojima, and Torishima in 2018 and describe them as a new species of catshark, Scyliorhinus hachijoensis. 
Morphological data on $S$. hachijoensis were obtained from fresh specimens that had been captured as bycatch in Splendid alfonsino (Beryx splendens Lowe, 1834) longline fishery in the waters around Mikurajima Is. $(n=$ 10), Hachijojima Is. $(n=18)$, and Torishima Is. $(n=3)$ (7 males and 24 females). Specimens of Scyliorhinus torazame from several catch localities were also examined as comparative material $(n=124 ; 57$ males and 67 females, see APPENDIX. 1). The holotype and paratypes were deposited in MSM (Marine Science Museum, Tokai University, Shizuoka, Japan), NSMT (National Museum of Nature and Science, Zoology Department, Division of Fishes, Tsukuba, Ibaraki, Japan), and SPMN (Museum of Natural and Environmental History, Shizuoka, Japan), and comparative specimens were examined from the following collections: ASIZ (Academia Sinica, Biodiversity Research Museum, Taipei), BSKU (Kochi University, Department of Natural Science, Faculty of Science, Kochi, Japan), FAKU (Kyoto University, Department of Bioresource Science, Faculty of Agriculture, Kyoto, Japan), HUMZ (Hokkaido University, Laboratory of Marine Zoology, Faculty of Fisheries, The Hokkaido University Museum, Hakodate, Hokkaido, Japan), KPM (Kanagawa Prefectural Museum of Natural History, Odawara, Kanagawa, Japan), MSM, NMMB (Pisces Collection, National Museum of Marine Biology and Aquarium, Pingtung, Taiwan), NSMT, SNFR (Seikai National Fisheries Research Institute, Nagasaki, Japan), and SPMN. Abbreviations were based on Fricke \& Eschmeyer (2020). We collected a total of 64 external measurements for males and 62 for females based on Springer (1964) and Compagno (2001), respectively, and these are expressed as percentages of total length (\%TL). The terminology used for color pattern followed Soares \& de Carvalho (2019). Clasper terminology was based on Compagno (1988), Soares \& de Carvalho (2019) and Soares (2020). The left clasper was used to study external anatomy and the right clasper to study internal anatomy. Counts of vertebrae were taken from radiographs obtained by using a SOFTEX M-60 (SOFTEX, Tokyo, Japan) at $25 \mathrm{kVp}$ and $2.5 \mathrm{~mA}$, for $3 \mathrm{sec}$. Tooth row counts and the nomenclature for dentition and dermal denticles followed Herman et al. (1993) and Compagno (1988). Dermal denticles were taken from the left side of the body above the pectoral fin and below the first and second dorsal fins. Photographs were taken with a Nikon D5300 digital camera and edited in the GNU Image Manipulation Program. Dermal denticles were photographed using a scanning electron microscope (JEOL. JSM-6390).

Methods for egg case measurement and terminology were based on Bustamante et al. (2013). Photographs were taken with a stereomicroscope (OLYMPUS SZX7) and Nikon D5300 digital camera and edited in GNU Image Manipulation Program.

The map showing the distribution of the new species was created using PanMap ver. 0.9.6 (Diepenbroek et al., 2000).

We compared nucleotide sequences of $S$. hachijoensis and $S$. torazame across three mitochondrial DNA regions: the 16S rRNA gene region (16S), cytochrome c oxidase subunit I (COI), and cytochrome $b$ (Cytb). Sequences from S. canicula (Linnaeus, 1758), Cephaloscyllium umbratile Jordan \& Fowler, 1903, and Poroderma pantherinum (Müller \& Henle, 1838) were used as outgroups (GenBank accession numbers: Y16067, NC 001950, KT 003686, NC 029399, and NC 043830). Muscle tissue samples were taken from 18 specimens of $S$. hachijoensis and nine specimens of $S$. torazame and preserved in $80 \%$ ethanol at $-30{ }^{\circ} \mathrm{C}$. Genomic DNA was extracted using the DNeasy Blood \& Tissue Kit (QUIAGEN, Hilden, Germany). PCR primers for the $16 \mathrm{~S}$ and Cytb gene regions were newly designed in the present study. The 16S primers were made from the complete mitochondrial genome sequences of 14 species of fishes; their GenBank accession numbers are as follows: AB032554 (Sardinops melanostictus (Temminck \& Schlegel, 1846)), AB034825 (Diplophos sp.), AB282840 (Himantolophus groenlandicus Reinhardt, 1837), AM489717 (Melanogrammus aeglefinus (Linnaeus, 1758)), AP002916 (Ateleopus japonicus Bleeker, 1854), AP002930 (Mugil cephalus Linnaeus, 1758), AP002952 (Stephanolepis cirrhifer (Temminck \& Schlegel, 1850)), AP004440 (Dactyloptena tiltoni Eschmeyer, 1997), AP004450 (Petroscirtes breviceps (Valenciennes, 1836)), AP006017 (Amphiprion ocellaris Cuvier, 1830), AP006238 (Mola mola (Linnaeus, 1758)), AP009227 (Rudarius ercodes Jordan \& Fowler, 1902), EU082205 (Halichoeres tenuispinis (Günther, 1862)), and GU452728 (Sebastiscus marmoratus (Cuvier, 1829)). Cytb primers were made from the complete mitochondrial genome sequences of S. canicula, C. umbratile, and Halaelurus buergeri (Müller \& Henle, 1838) (GenBank accession numbers Y16067, KT003686, and KU892589, respectively). The primer sequences for 16S were univ1952_16SF (5'TCGCCTGTTTACCAAAAACA-3') and univ2571_16SR (5'-CTCCGGTCTGAACTCAGATC-3'), and those for Cytb were SchCytbF (5'-TTTATGGCCACAAATAYCCG-3') and SchCytbR (5'-CAGCTGATGAGAGGTATCAC3'). For the COI region, we used the primers VF2_t1, FishF2_t1, FishR2_t1, and FR1d_t1 (Ivanova et al. 2007). 
PCR amplification was carried out with the following $10 \mu \mathrm{L}$ reaction mixture: $4.95 \mu \mathrm{L}$ of distilled water, $1 \mu \mathrm{L}$ of $10 \times$ EX buffer, $1 \mu \mathrm{L}$ of dNTPs $(2.5 \mathrm{mM}$ each $), 1 \mu \mathrm{L}$ of each primer $(10 \mu \mathrm{M}), 0.05 \mu \mathrm{L}$ of EX Taq polymerase (Takara Bio Inc. Shiga, Japan), and $1 \mu \mathrm{L}$ of genomic DNA (approx. 10-50 ng). The PCR procedure was as follows: an initial denaturation step at $95^{\circ} \mathrm{C}$ for $2 \mathrm{~min}$, denaturation at $95{ }^{\circ} \mathrm{C}$ for $30 \mathrm{sec}$, annealing at $55^{\circ} \mathrm{C}$ for $40 \mathrm{sec}$, and extension at $72{ }^{\circ} \mathrm{C}$ for $1 \mathrm{~min}\left(1.5 \mathrm{~min}\right.$ for Cytb), followed by 30 cycles of $95^{\circ} \mathrm{C}$ for $30 \mathrm{sec}, 55^{\circ} \mathrm{C}$ for $40 \mathrm{sec}$, and 72 ${ }^{\circ} \mathrm{C}$ for $1 \mathrm{~min}$, with a final extension at $72{ }^{\circ} \mathrm{C}$ for $5 \mathrm{~min}$, after which samples were maintained at $4{ }^{\circ} \mathrm{C}$. For nucleotide sequencing, we used the BigDye Terminator ver. 3.1 Cycle Sequencing Kit (Applied Biosystems, Inc. California, USA). The reaction profile consisted of an initial denaturation step at $95^{\circ} \mathrm{C}$ for $2 \mathrm{~min}$, and then denaturation at $95{ }^{\circ} \mathrm{C}$ for $10 \mathrm{sec}$, annealing at $50^{\circ} \mathrm{C}$ for $5 \mathrm{sec}$, and extension at $60^{\circ} \mathrm{C}$ for $2.5 \mathrm{~min}$, followed by 40 cycles of $95^{\circ} \mathrm{C}$ for $10 \mathrm{sec}$, $50{ }^{\circ} \mathrm{C}$ for $5 \mathrm{sec}$, and $60^{\circ} \mathrm{C}$ for $2.5 \mathrm{~min}$, after which samples were maintained at $4{ }^{\circ} \mathrm{C}$. The sequencing primers were univ1952_16SF (for 16S), SchCytbF (for Cytb), and M13F (-21) (for COI; Ivanova et al. 2007). DNA sequences were aligned in MEGA-X ver. 10.0.5 (Kumar et al. 2018). Sequence divergences were calculated by using the Tamura-Nei model (Tamura \& Nei 1993) with a discrete gamma distribution. Maximum likelihood phylogenetic trees were constructed in MEGA-X. The robustness of the ML tree was assessed using the bootstrap method with 1000 replicates. All sequences were deposited online (GenBank accession numbers: LC517181 to LC517261 for $S$. hachijoensis and S. torazame, see APPENDIX 2).

\section{Scyliorhinus hachijoensis sp. nov.}

(Table 1 and Figs. 1-9)

New English name: Cinder cloudy catshark; new Japanese name: Fukami-torazame.

Scyliorhinus sp.: Hagiwara, 1993: 1, 9, Tables 1-3, Fig. 3 (keeping and reproduction)

\section{Holotype.}

NSMT-P135960, adult male, $370 \mathrm{~mm}$ TL (off the east coast of Hachijojima Island, Tokyo, Japan, 200-300 m depth, 24 October 2018).

\section{Paratypes.}

MSM-19-292, female, $331 \mathrm{~mm}$ TL (off the east coast of Hachijojima Island, Tokyo, Japan, 300-400 m depth, 12 July 2018); MSM-19-293, female, 354 mm TL (off the east coast of Hachijojima Island, Tokyo, Japan, 300400 m depth, 12 July 2018); MSM-19-294, female, 326 mm TL (off the east coast of Hachijojima Island, Tokyo, Japan, 300-400 m depth, 12 July 2018); MSM-19-295, female, $354 \mathrm{~mm}$ TL (off the east coast of Hachijojima Island, Tokyo, Japan, 300-400 m depth, 12 July 2018); MSM-19-296, female, $368 \mathrm{~mm}$ TL (off the east coast of Hachijojima Island, Tokyo, Japan, 300-400 m depth, 12 July 2018); MSM-19-297, female, $382 \mathrm{~mm}$ TL (off the east coast of Hachijojima Island, Tokyo, Japan, 300-400 m depth, 12 July 2018); MSM-19-298, female, 366 mm TL (off the east coast of Hachijojima Island, Tokyo, Japan, 200-300 m depth, 18 August 2018); MSM-19-299, female, $350 \mathrm{~mm}$ TL (off the east coast of Hachijojima Island, Tokyo, Japan, 200-300 m depth, 18 August 2018); MSM19-300, female, $356 \mathrm{~mm}$ TL (off the east coast of Hachijojima Island, Tokyo, Japan, 200-300 m depth, 18 August 2018); MSM-19-301, female, 368 mm TL (off the east coast of Hachijojima Island, Tokyo, Japan, 200-300 m depth, 18 August 2018); NSMT-P 135961, female, $322 \mathrm{~mm}$ TL (off the east coast of Hachijojima Island, Tokyo, Japan, 400-500 m depth, 12 July 2018); NSMT-P 135962, female, $348 \mathrm{~mm}$ TL (off the east coast of Hachijojima Island, Tokyo, Japan, $500 \mathrm{~m}$ depth, 11 July 2018); NSMT-P 135963, male, $384 \mathrm{~mm}$ TL (off the east coast of Mikurajima Island, Tokyo, Japan, 100-200 m depth, 24 October 2018); NSMT-P 135964, female, $364 \mathrm{~mm}$ TL (off the east coast of Mikurajima Island, Tokyo, Japan, 100-200 m depth, 24 October 2018); NSMT-P 135965, male, 419 mm TL (off the east coast of Torishima Island, Tokyo, Japan, 600-650 m depth, 27 June 2018); NSMT-P 136476, female, 386 $\mathrm{mm}$ TL (off the east coast of Hachijojima Island, Tokyo, Japan, $300 \mathrm{~m}$ depth, 15 November 2018); NSMT-P 136477, female, $342 \mathrm{~mm}$ TL (off the east coast of Mikurajima Island, Tokyo, Japan, 100-200 m depth, 24 October 2018); NSMT-P 136478, male, $346 \mathrm{~mm}$ TL (off the east coast of Mikurajima Island, Tokyo, Japan, 100-200 m depth, 24 October 2018); SPMN-PI 45542, female, $370 \mathrm{~mm}$ TL (off the east coast of Hachijojima Island, Tokyo, Japan, $300 \mathrm{~m}$ depth, 15 November 2018); SPMN-PI 45543, female, $364 \mathrm{~mm}$ TL (off the east coast of Hachijojima Island, Tokyo, Japan, $300 \mathrm{~m}$ depth, 15 November 2018); SPMN-PI 45544, female, $345 \mathrm{~mm}$ TL (off the east coast of Hachijojima 
Island, Tokyo, Japan, $300 \mathrm{~m}$ depth, 15 November 2018); SPMN-PI 45545, female, $372 \mathrm{~mm}$ TL (off the east coast of Hachijojima Island, Tokyo, Japan, $300 \mathrm{~m}$ depth, 15 November 2018); SPMN-PI 45546, female, $301 \mathrm{~mm}$ TL (off the east coast of Mikurajima Island, Tokyo, Japan, 100-200 m depth, 24 October 2018); SPMN-PI 45547, female, $355 \mathrm{~mm}$ TL (off the east coast of Mikurajima Island, Tokyo, Japan, 100-200 m depth, 24 October 2018); SPMN-PI 45548, female, $305 \mathrm{~mm}$ TL (off the east coast of Mikurajima Island, Tokyo, Japan, 100-200 m depth, 24 October 2018); SPMN-PI 45549, male, $294 \mathrm{~mm}$ TL (off the east coast of Mikurajima Island, Tokyo, Japan, 100-200 m depth, 24 October 2018); SPMN-PI 45550, male, $335 \mathrm{~mm}$ TL (off the east coast of Mikurajima Island, Tokyo, Japan, 100-200 m depth, 24 October 2018); SPMN-PI 45551, female, $356 \mathrm{~mm}$ TL (off the east coast of Mikurajima Island, Tokyo, Japan, 100-200 m depth, 24 October 2018).

\section{Other materials.}

HUMZ113575, female, 353 mm TL (off Torishima Island, Tokyo, Japan); HUMZ113576, male, 372 mm TL (off Torishima Island, Tokyo, Japan).

\section{Diagnosis.}

A species of Scyliorhinus distinguished by its anterior nasal flaps not reaching the upper lip (vs. flaps reaching upper lip, and sometimes covering it, in S. canicula, S. cervigoni, S. comoroensis, S. duhamelii, S. garmani and S. stellaris); nasoral grooves absent and posterior nasal flaps situated posterior to excurrent apertures (vs. nasoral grooves prexents and posterior nasal flaps laterally situated in S. canicula and S. duhamelii); mouth length less than half of mouth width (vs. mouth length more than or equal half of mouth width except in S. torazame and S. ugoi); anal fin height more than caudal peduncle height (vs. less than caudal peduncle height in S.boa, S. duhamelii, S. torazame and $S$. torrei), and greater than or equal to half of mouth width (vs. less than half of mouth width in $S$. boa, S. capensis, S. duhamelii, S. haeckelii, S. hesperius, S.meadi, S. torazame, S. torrei and S. ugoi); saddles darker than the background color (vs. inconspicuous or absent in S. boa, S. cabofriensis, S. cervigoni, S. duhamelii, $S$. garmani and S. torrei, and dark lines in S. retifer); body grayish brown to dark brown with well-defined light spots and small dark spots (vs. spots absent in S. retifer, yellow to golden spots in $S$. capensis, light spots absent in $S$. cervigoni, S. garmani, S. meadi and S. retifer, and dark spots absent in S. capensis, S. comoroensis, S. hesperius, S. meadi, S. torazame and $S$. torrei); light spots spiracle-sized or larger (vs. predominantly smaller than spiracles in S. boa, S. cabofriensis, S. canicula, S. duhamelii, S. stellaris and S. ugoi); dark spots smaller than spiracles (vs. predominantly larger than spiracles in S. cervigoni, S. duhamelii, S. garmani, S. haeckelii and S. stellaris); number of monospondylous vertebrae 34-36 (vs. counts higher except in S. duhamelii, S. torazame and S. torrei); clasper with hooks (vs. absent in all other species except $S$. torazame); accessory terminal cartilage present (vs. absent in S. cabofriensis, S. cervigoni, S. comoroensis, S. duhamelii, S. haeckelii, S. stellaris, S. torrei and S. ugoi); egg case surface with irregularities (vs. smooth in all other species).

\section{Description.}

Morphometric measurements are given in Table 1.

Body slender and tapering to caudal fin (Fig. 1), precaudal length $77.3 \% \mathrm{TL}$ (72.0-76.4 \% TL in palatypes). Prepectoral length 0.4 times prepelvic length. Pectoral-pelvic space 1.5 times pelvic-anal space (1.7-2.5 times in female, 1.4-1.9 times in male). Interdorsal space 1.6 times dorsal-caudal space. Trunk shorter than tail; snout-vent length $44.1 \% \mathrm{TL}$ ( $42.0-44.6 \% \mathrm{TL})$. First dorsal origin above insertion of pelvic fin, second dorsal origin forward to anal insertion. No interdorsal, postdorsal, or postanal ridges; lateral crest on caudal peduncle absent.

Head (Fig. 2) moderately broad and depressed. Head length (HL) $18.9 \%$ TL (17.2-19.6 \%TL) and 1.4 times head width. Snout short, prenasal length $2.3 \% \mathrm{TL}(2.1-2.6 \% \mathrm{TL})$ and 0.6 times preoral length. Preoral length 3.8 \%TL (3.2-4.4\%TL), 0.4 times mouth width and 0.9 times preorbital length. Preorbital length $4.1 \% \mathrm{TL}$ (4.1-5.1 $\% \mathrm{TL}), 0.5$ times interorbital space.

Eyes (Fig. 2A) large and slitlike, eye length $4.1 \% \mathrm{TL}$ (3.3-4.4\%TL), 0.2 times head length, with lower edges medial to horizontal head rim in dorsal view; subocular ridge strong. Nictitating lower eyelid of rudimentary type, with shallow subocular pocket and secondary lower eyelid free from upper eyelid. Eye length 5.3 times and interorbital distance 9.6 times spiracle length.

Spiracles (Fig. 2A) close behind but clearly separated from eyes, positioned dorsolaterally on head and somewhat lower than level of eye notch. 
TABLE 1. Morphometric and meristic data for Scyliorhinus hachijoensis $(\mathrm{n}=31)$ and S. torazame $(\mathrm{n}=124)$. SD, Standard deviation; n, number of examined specimens. Total length (TL) is reported in mm, other measurements are shown as percentages of TL. The number of specimens for spiral valves is 18 in each species, and for vertebral counts is 11 S. hachijoensis and 23 S. torazame. "this study, ${ }^{\dagger}$ Soares \& de Carvalho (2019).

\begin{tabular}{|c|c|c|c|c|c|c|c|c|}
\hline \multirow{3}{*}{ Measurements } & \multicolumn{4}{|c|}{ Scyliorhinus hachijoensis } & \multicolumn{4}{|c|}{ Scyliorhinus torazame } \\
\hline & \multirow{2}{*}{ Holotype } & \multicolumn{3}{|c|}{ Paratypes } & \multirow{2}{*}{ Range $^{\dagger}$} & \multirow{2}{*}{ Mean $^{\dagger}$} & \multirow{2}{*}{$\mathrm{SD}^{\dagger}$} & \multirow{2}{*}{ Range $^{\dagger \dagger}$} \\
\hline & & Range & Mean & SD & & & & \\
\hline Total length (TL) & 370 & $294-419$ & 353 & 27 & $87-465$ & 357 & 94 & $95.8-778.0$ \\
\hline Precaudal length & 77.3 & $72.0-76.4$ & 74.8 & 1.0 & $66.7-79.4$ & 73.8 & 2.5 & $68.2-78.2$ \\
\hline Prenasal length & 2.3 & $2.1-2.6$ & 2.4 & 0.2 & $1.8-3.8$ & 2.4 & 0.3 & $1.6-4.1$ \\
\hline Preoral length & 3.8 & $3.2-4.4$ & 4.0 & 0.3 & $3.2-5.6$ & 4.0 & 0.4 & $3.4-5.9$ \\
\hline Preorbital length & 4.1 & $4.1-5.1$ & 4.6 & 0.2 & $3.2-5.8$ & 4.4 & 0.5 & $5.3-7.8$ \\
\hline Prespiracular length & 8.8 & $7.9-9.6$ & 8.7 & 0.4 & $7.3-10.7$ & 8.5 & 0.6 & $8.4-12.4$ \\
\hline Prebranchial length & 14.1 & $12.5-15.3$ & 13.7 & 0.7 & $11.9-17.2$ & 13.5 & 0.9 & $12.7-17.7$ \\
\hline Head length (HL) & 18.9 & $17.2-19.6$ & 18.4 & 0.6 & $15.9-21.8$ & 18.1 & 0.9 & $17.1-22.4$ \\
\hline Prepectoral length & 16.5 & $14.4-17.6$ & 15.9 & 0.7 & $13.8-19.5$ & 15.7 & 0.9 & $15.5-20.5$ \\
\hline Prepelvic length & 38.4 & $37.7-40.1$ & 39.2 & 0.7 & $35.0-43.6$ & 39.1 & 1.7 & $36.2-45.3$ \\
\hline Snout-vent length & 44.1 & $42.0-44.6$ & 43.4 & 0.7 & $36.9-46.6$ & 43.1 & 1.9 & $40.4-50.1$ \\
\hline Preanal length & 60.3 & $55.1-59.2$ & 57.5 & 0.9 & $52.7-62.5$ & 59.0 & 1.9 & - \\
\hline Pre-first dorsal length & 48.9 & $46.8-50.3$ & 48.1 & 1.0 & $43.4-53.2$ & 48.7 & 1.9 & $43.0-51.9$ \\
\hline Pre-second dorsal length & 65.7 & $62.6-65.8$ & 64.3 & 1.0 & $57.5-68.9$ & 65.0 & 2.1 & - \\
\hline Interdorsal distance & 9.7 & $8.1-11.3$ & 9.3 & 0.8 & $7.1-11.8$ & 9.3 & 0.9 & $7.5-12.3$ \\
\hline Dorsal-caudal distance & 6.2 & $3.3-6.1$ & 4.9 & 0.7 & $0.8-6.5$ & 3.4 & 1.5 & $1.1-5.9$ \\
\hline Pectoral-pelvic distance & 16.6 & $14.9-19.8$ & 17.0 & 0.9 & $9.3-21.9$ & 16.6 & 2.1 & $11.7-20.2$ \\
\hline Pelvic anal distance & 11.0 & $6.9-11.8$ & 8.4 & 1.0 & $7.1-14.2$ & 9.8 & 1.2 & $5.9-14.5$ \\
\hline Anal caudal distance & 7.0 & $5.6-8.1$ & 6.9 & 0.6 & $4.3-9.6$ & 7.2 & 1.0 & $5.2-9.7$ \\
\hline Interorbital distance & 7.5 & $5.6-8.0$ & 7.4 & 0.5 & $6.0-8.6$ & 7.3 & 0.5 & $5.7-9.2$ \\
\hline Internarial distance & 2.2 & $1.5-2.2$ & 1.9 & 0.2 & $1.3-3.2$ & 1.9 & 0.3 & $4.8-8.5$ \\
\hline Mouth width & 8.6 & $8.0-9.4$ & 8.6 & 0.4 & $6.7-9.4$ & 8.3 & 0.5 & $7.5-10.6$ \\
\hline Mouth length & 2.9 & $2.5-4.4$ & 3.0 & 0.4 & $2.3-4.7$ & 3.0 & 0.3 & $4.2-6.9$ \\
\hline Lower labial furrow length & 1.0 & $0.9-1.5$ & 1.2 & 0.2 & $0.4-1.4$ & 0.8 & 0.2 & $1.3-2.7$ \\
\hline Eye length & 4.1 & $3.3-4.4$ & 3.8 & 0.3 & $3.0-6.3$ & 3.9 & 0.4 & $2.9-7.5$ \\
\hline Spiracle length & 0.8 & $0.5-0.9$ & 0.6 & 0.1 & $0.3-1.0$ & 0.7 & 0.1 & $0.6-2.1$ \\
\hline Eye-spiracle length & 0.7 & $0.4-0.7$ & 0.6 & 0.1 & $0.2-0.9$ & 0.4 & 0.1 & $0.8-3.4$ \\
\hline First gill slit height & 2.2 & $1.5-2.3$ & 2.0 & 0.2 & $0.8-2.7$ & 1.7 & 0.3 & $1.6-3.3$ \\
\hline Fifth gill slit height & 1.4 & $0.7-1.6$ & 1.1 & 0.2 & $0.4-1.3$ & 0.8 & 0.2 & $0.7-1.7$ \\
\hline Head width & 13.2 & $11.5-13.3$ & 12.5 & 0.4 & $10.9-14.3$ & 12.7 & 0.7 & $10.6-14.8$ \\
\hline Pectoral base & 7.0 & $6.1-8.0$ & 6.9 & 0.5 & $6.2-8.8$ & 7.2 & 0.5 & $5.5-8.8$ \\
\hline
\end{tabular}

...... Continued on the next page 
TABLE 1. (Continued)

\begin{tabular}{|c|c|c|c|c|c|c|c|c|}
\hline \multirow{3}{*}{ Measurements } & \multicolumn{4}{|c|}{ Scyliorhinus hachijoensis } & \multicolumn{4}{|c|}{ Scyliorhinus torazame } \\
\hline & \multirow{2}{*}{ Holotype } & \multicolumn{3}{|c|}{ Paratypes } & \multirow{2}{*}{ Range $^{\dagger}$} & \multirow{2}{*}{ Mean $^{\dagger}$} & \multirow{2}{*}{$\mathrm{SD}^{\dagger}$} & \multirow{2}{*}{ Range $^{\dagger \dagger}$} \\
\hline & & Range & Mean & SD & & & & \\
\hline Pectoral anterior margin & 16.5 & $14.0-17.1$ & 15.6 & 0.7 & $10.6-16.2$ & 13.2 & 1.1 & $11.7-17.0$ \\
\hline Pectoral posterior margin & 10.9 & $9.1-11.5$ & 10.3 & 0.5 & $5.6-11.0$ & 8.7 & 1.0 & $5.8-10.1$ \\
\hline Pectoral inner margin & 5.1 & $4.2-6.0$ & 5.0 & 0.5 & $3.4-6.4$ & 4.8 & 0.6 & $4.7-7.8$ \\
\hline Pelvic length & 19.1 & $11.2-20.8$ & 13.3 & 2.9 & $9.3-22.5$ & 14.8 & 3.8 & $10.4-22.0$ \\
\hline Pelvic base & 11.1 & $9.3-10.7$ & 10.0 & 0.4 & $7.4-13.5$ & 10.1 & 1.0 & $6.9-13.7$ \\
\hline Pelvic anterior margin & 9.7 & $8.2-10.7$ & 9.0 & 0.5 & $5.1-9.6$ & 7.5 & 0.9 & $6.3-10.7$ \\
\hline Pelvic inner margin & 8.6 & $1.5-11.1$ & 3.3 & 2.9 & $0.9-11.2$ & 4.9 & 3.3 & $2.1-12.2$ \\
\hline Clasper inner length & 14.1 & $6.8-17.2$ & 13.8 & 4.1 & $3.6-17.3$ & 12.2 & 4.7 & $3.6-17.1$ \\
\hline Clasper outer length & 9.0 & $1.4-12.3$ & 8.8 & 4.4 & $0.4-12.1$ & 7.5 & 4.1 & $1.5-13.2$ \\
\hline Anal length & 10.7 & $10.1-12.9$ & 11.3 & 0.6 & $9.1-11.8$ & 10.3 & 0.6 & - \\
\hline Anal base & 8.1 & $7.9-11.0$ & 9.1 & 0.6 & $6.8-9.7$ & 8.0 & 0.5 & $4.1-11.1$ \\
\hline Anal anterior margin & 8.0 & $7.5-9.3$ & 8.7 & 0.4 & $6.1-9.3$ & 7.6 & 0.6 & $7.1-11.5$ \\
\hline Anal posterior margin & 5.3 & $4.9-6.9$ & 5.8 & 0.5 & $2.3-5.8$ & 4.5 & 0.6 & $3.6-5.7$ \\
\hline Anal inner margin & 2.4 & $1.7-2.6$ & 2.2 & 0.2 & $0.9-3.0$ & 2.3 & 0.3 & $1.9-5.1$ \\
\hline Anal height & 4.3 & $4.0-5.3$ & 4.7 & 0.3 & $2.3-4.1$ & 3.2 & 0.4 & $3.1-7.7$ \\
\hline First dorsal length & 10.6 & $9.5-11.5$ & 10.4 & 0.5 & $8.0-11.8$ & 10.0 & 0.6 & - \\
\hline First dorsal base & 7.3 & $6.4-8.0$ & 7.2 & 0.4 & $6.0-9.7$ & 7.3 & 0.6 & $6.9-10.8$ \\
\hline First dorsal anterior margin & 10.9 & $10.0-12.6$ & 11.0 & 0.6 & $8.0-11.3$ & 9.5 & 0.6 & $8.6-12.6$ \\
\hline First dorsal posterior margin & 5.9 & $5.3-6.6$ & 6.1 & 0.3 & $3.3-6.6$ & 5.1 & 0.7 & - \\
\hline First dorsal inner margin & 2.5 & $1.9-3.0$ & 2.2 & 0.3 & $1.6-3.2$ & 2.3 & 0.3 & $1.7-3.8$ \\
\hline First dorsal height & 6.4 & $6.0-7.2$ & 6.8 & 0.3 & $3.6-6.7$ & 5.4 & 0.6 & $4.8-7.8$ \\
\hline Second dorsal length & 8.4 & $7.5-9.0$ & 8.4 & 0.4 & $6.6-9.6$ & 8.0 & 0.5 & - \\
\hline Second dorsal base & 5.8 & $5.1-6.5$ & 5.9 & 0.4 & $4.6-7.3$ & 5.5 & 0.5 & $5.2-7.9$ \\
\hline Second dorsal anterior margin & 7.6 & $6.9-8.5$ & 7.8 & 0.4 & $5.1-7.9$ & 6.6 & 0.5 & $6.1-9.4$ \\
\hline $\begin{array}{l}\text { Second dorsal posterior } \\
\text { margin }\end{array}$ & 4.5 & $3.7-4.6$ & 4.1 & 0.3 & $2.0-4.6$ & 3.5 & 0.5 & - \\
\hline Second dorsal inner margin & 2.3 & $2.0-2.7$ & 2.3 & 0.2 & $1.3-3.0$ & 2.4 & 0.3 & $1.6-3.1$ \\
\hline Second dorsal height & 4.2 & $3.6-4.6$ & 4.1 & 0.3 & $2.1-4.2$ & 3.3 & 0.4 & $2.8-5.0$ \\
\hline Dorsal caudal margin & 22.5 & $23.3-26.9$ & 24.9 & 0.8 & $21.4-33.0$ & 26.1 & 2.3 & $21.8-31.8$ \\
\hline Preventral caudal margin & 10.8 & $10.4-13.2$ & 11.5 & 0.7 & $8.9-17.5$ & 11.3 & 1.1 & $8.8-17.7$ \\
\hline Terminal caudal margin & 6.4 & $4.8-6.8$ & 5.9 & 0.5 & $3.1-7.0$ & 5.3 & 0.7 & $3.3-6.3$ \\
\hline Subterminal caudal margin & 3.8 & $3.7-5.6$ & 4.3 & 0.4 & $2.7-6.1$ & 4.0 & 0.5 & $2.8-6.8$ \\
\hline Caudal peduncle height & 3.0 & $2.4-3.4$ & 3.0 & 0.2 & $2.5-4.4$ & 3.2 & 0.3 & $3.1-4.8$ \\
\hline Caudal peduncle width & 2.3 & $1.8-2.7$ & 2.2 & 0.2 & $1.7-3.6$ & 2.7 & 0.3 & $2.2-3.9$ \\
\hline
\end{tabular}

...... Continued on the next page 
TABLE 1. (Continued)

\begin{tabular}{|c|c|c|c|c|c|c|c|c|}
\hline \multirow{3}{*}{ Measurements } & \multicolumn{4}{|c|}{ Scyliorhinus hachijoensis } & \multicolumn{4}{|c|}{ Scyliorhinus torazame } \\
\hline & \multirow{2}{*}{ Holotype } & \multicolumn{3}{|c|}{ Paratypes } & \multirow{2}{*}{ Range $^{\dagger}$} & \multirow{2}{*}{ Mean $^{\dagger}$} & \multirow{2}{*}{$\mathrm{SD}^{\dagger}$} & \multirow{2}{*}{ Range $^{\dagger \dagger}$} \\
\hline & & Range & Mean & SD & & & & \\
\hline \multicolumn{9}{|l|}{ Meristics } \\
\hline Upper tooth rows & 39 & $36-49$ & - & - & $34-57$ & - & - & $50-76$ \\
\hline Lower tooth rows & 36 & $32-43$ & - & - & $34-52$ & - & - & $45-81$ \\
\hline Spiral valves & 8 & $7-8$ & - & - & $6-7$ & - & - & $6-7$ \\
\hline Monospondylous vertebrae & 34 & $34-36$ & - & - & $34-37$ & - & - & $32-37$ \\
\hline Precaudal vertebrae & 77 & $71-78$ & - & - & $66-77$ & - & - & - \\
\hline Total vertebrae & 112 & $109-115$ & - & - & $102-112$ & - & - & $105-126$ \\
\hline
\end{tabular}

First and second gill openings about equal in height, with last three tapering down to fifth (Fig. 2A, B). First gill opening height 1.6 times that of fifth. All gill openings slightly concave and not elevated on dorsolateral surface of head. Gill filaments not visible externally.

Nostrils (Fig. 2C) with broad incurrent apertures, without nasoral grooves or nasal barbels and with small and oval excurrent apertures. Anterior nasal flaps large, triangular, and covering posterior nasal flap and excurrent aperture, extending just anterior to mouth, close to but not touching upper lip. Mesonarial ridge distinct but not exceeding posterior border of anterior nasal flap. Posterior nasal flaps small and rectangular, situated on posterior border of excurrent aperture. Internarial distance 0.3 times interorbital distance.

Mouth (Fig. 2A, C) arched, moderately wide, and short. Mouth length $2.9 \% \mathrm{TL}(2.5-4.4 \% \mathrm{TL}), 0.3$ times mouth width and 0.2 times head length; mouth width 0.7 times head width. Upper labial furrows absent. Lower labial furrows short and narrow, length 0.1 times mouth width.

Tooth counts 39/36 (36-49/32-43 in paratypes); teeth morphologically similar in both jaws. Monognathic heterodonty gradual and well developed; anterolateral teeth abruptly larger than symphysial ones and smaller distally; posterior teeth with smaller principal cusps than anterolateral teeth (Fig. 3). Sexual heterodonty weak. Symphysial teeth with principal cusp and one weak cusplet on each side. Anterolateral teeth with more developed principal cusp than symphysial teeth and two to four cusplets; most outside cusplets small and undeveloped when with three or four cusplets. Posterior teeth have two or three cusplets and tilted to the outside; principal cusp weakly developed.

Pectoral fins (Figs. 1, 4A, B) large and rounded-triangular, not falcate, with broadly convex anterior margins and almost straight posterior and inner margins. Pectoral fin inner corner obtuse angle $\left(96^{\circ}\right.$ in holotype, $90^{\circ}-102^{\circ}$ in paratypes). Pectoral origin under third gill opening, with base length 0.8 times mouth width. Pectoral anterior margin 2.4 times base length and 1.5 times posterior margin.

Pelvic fins (Figs. 1, 4A, B) broadly triangular, with rounded outer corner. Pelvic anterior margin 0.9 times base length. In males, pelvic inner margins fused together forming a pelvic apron, elongated posteriorly and covering claspers. In females, the pelvic fin apex located obviously posterior to middle of fin, and angle of pelvic fin inner corner $63^{\circ}-75^{\circ}$ in paratypes.

Claspers (Fig. 5A) moderately long and cylindrical, extending beyond free rear tips of pelvic fins in adult specimens. Clasper inner length 14.1 \%TL (14.1-17.2 \%TL in mature males of paratypes), 1.4 times (1.4-1.8 times in mature males of paratypes) pelvic anterior margin and 1.6 times (1.4-1.7 times) clasper outer length. Most of clasper surface (except part of medial surface of clasper shaft and part of exorhipidion, rhipidion, and terminal dermal cover) covered by dermal denticles with anteriorly directed crowns. Clasper hooks present, forming row from behind apopyle to posterior tip of exorhipidion, running along medial border of exorhipidion (Fig. 5A, B). Rhipidion well developed, partly covered medially by prominent exorhipidion and anteriorly by cover rhipidion. Rhipidion insertion point located in anterior portion of dorsal terminal 2 cartilage and extending to end of glans. Cover rhipidion expanded medially, reaching exorhipidion with nearly straight border at the shaft and meandering border at glans. Both cover rhipidion and exorhipidion cover clasper groove. Envelope, pseudopera, and pseudosiphon absent. Terminal dermal cover smooth, and contacting exorhipidion and rhipidion. 

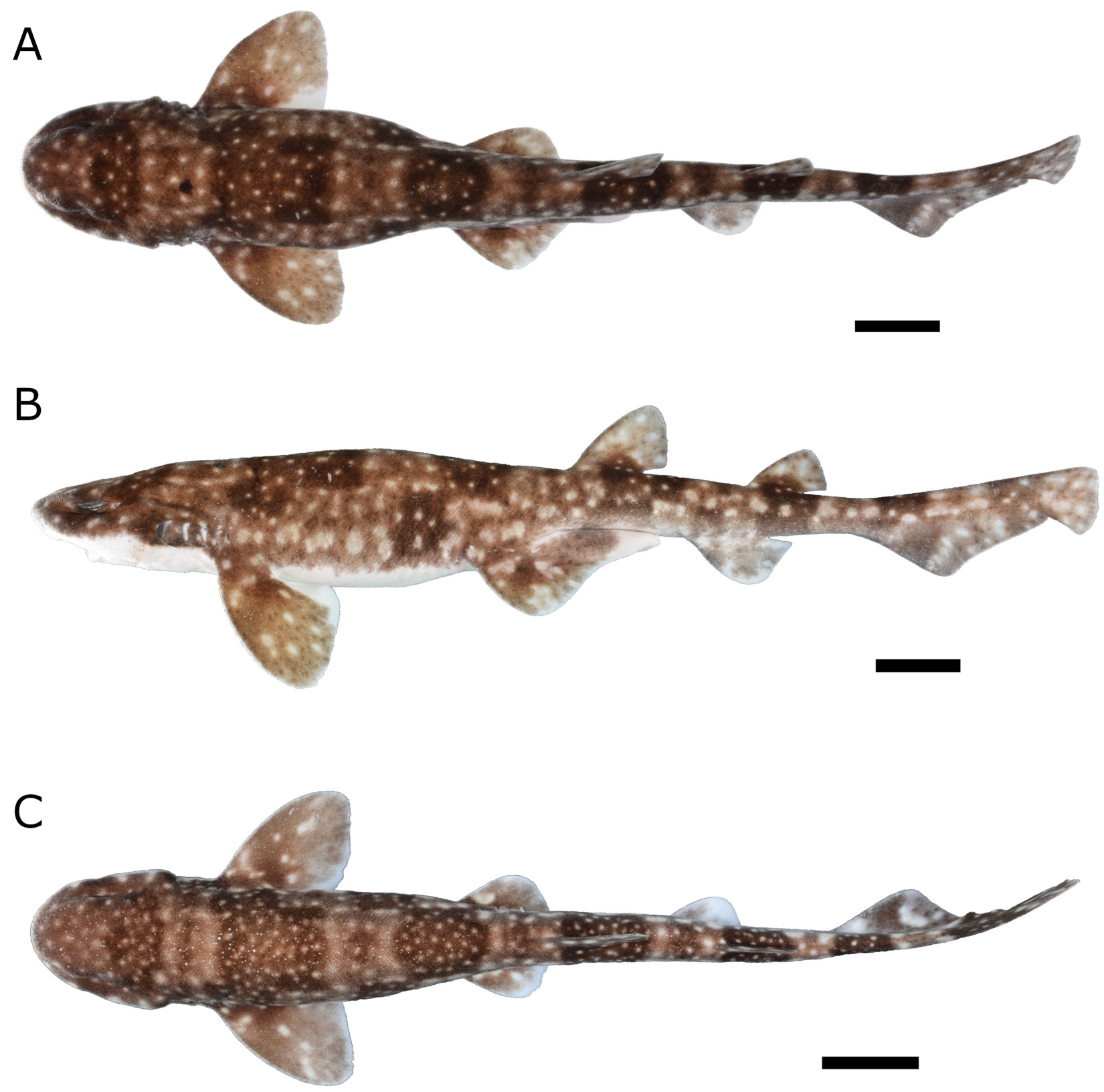

D

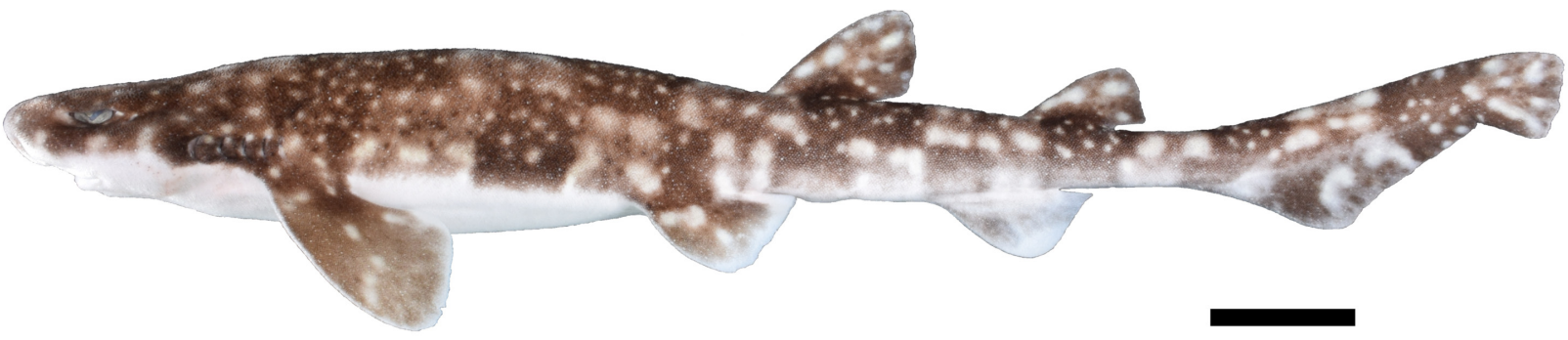

FIG. 1. Scyliorhinus hachijoensis, external morphology. (A, B) NSMT-P 135960, male, $370 \mathrm{~mm}$ TL (off the east coast of Hachijojima Island). (C, D) NSMT-P 135961, female, $322 \mathrm{~mm}$ TL (off the east coast of Hachijojima Island). Panels show (A, C) dorsal and $(\mathrm{B}, \mathrm{D})$ lateral views of the specimens. Scale bar $=30 \mathrm{~mm}$. 
A

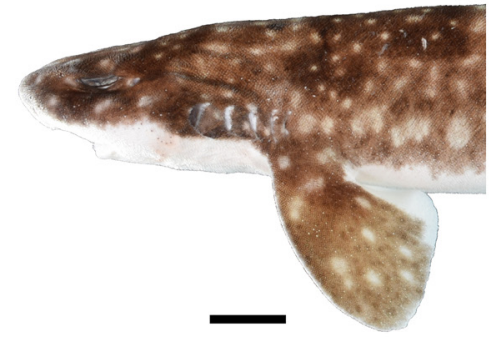

B

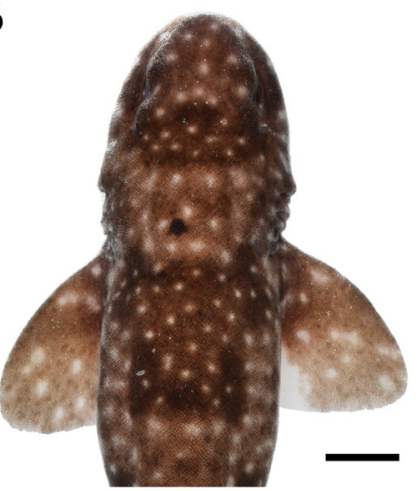

C

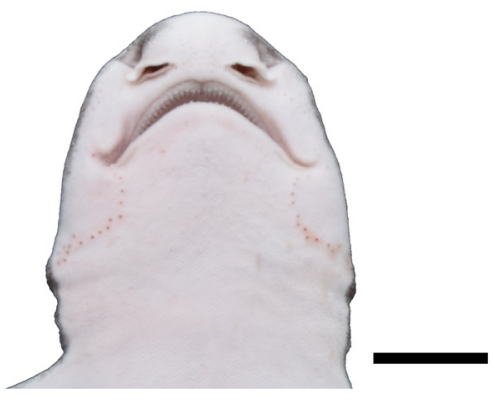

FIG. 2. Close-ups of the head of Scyliorhinus hachijoensis, NSMT-P 135960, male, $370 \mathrm{~mm}$ TL (off the east coast of Hachijojima Island). Panels show (A) lateral, (B) dorsal, and (C) ventral views. Scale bar $=20 \mathrm{~mm}$.

A

P

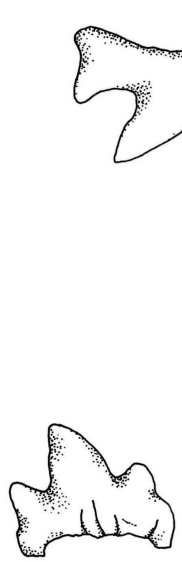

$\mathrm{P}$
AL

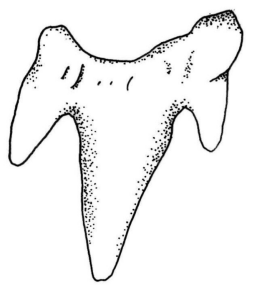

$\mathrm{AL}$

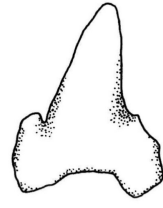

$\mathrm{S}$

B

$P$

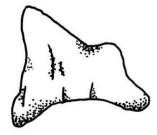

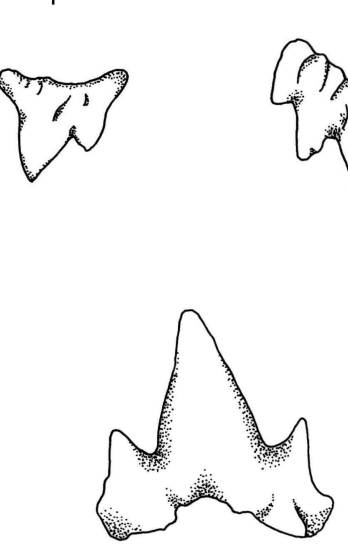

$\mathrm{AL}$
AL
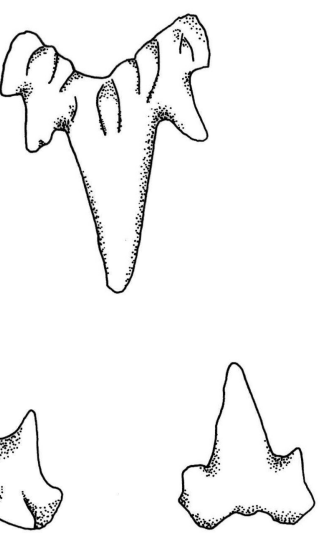

S

FIG. 3. Teeth of Scyliorhinus hachijoensis, labial view. (A) NSMT-P 135960, male, $370 \mathrm{~mm}$ TL (off the east coast of Torishima Island). (B) NSMT-P 135962, female, $348 \mathrm{~mm}$ TL (off the east coast of Hachijojima Island). AL, anterolateral tooth; P, posterior tooth; S, symphyseal. Scale bar $=0.5 \mathrm{~mm}$.

Clasper skeleton relatively simple (Fig. 5C). Ventral and dorsal terminal cartilage forms spoon-shaped structure; dorsal terminal begins anteriorly but ends together with ventral cartilage. Accessory terminal cartilage quadrangle situated posteriorly and inside ventral marginal cartilage. Dorsal terminal 2 cartilage beginning posteriorly and ventrally to dorsal marginal cartilage, elongated and rod-like, laterally positioned on dorsal terminal cartilage and extending throughout its length. Ventral terminal 2 cartilage elongated and oval, laterally and posteriorly positioned on ventral terminal cartilage and extending to half its length.

First dorsal fin (Fig. 1) sub-rectangular and triangular, not falcate, with nearly straight anterior margin, round apex, and angular free rear tip. First dorsal base 0.8 times the interdorsal space. First dorsal anterior margin length 1.5 times its base, and first dorsal height 0.9 times its base.

Second dorsal fin (Fig. 1) triangular, not falcate and smaller than first dorsal fin, with nearly straight anterior margin, straight posterior margin, rounded apex, and sharper free rear tip than first dorsal fin. Second dorsal fin base 1.4 times its height and 0.9 times dorsal-caudal distance. Second dorsal anterior margin 1.3 times its base. Second dorsal fin base 0.8 times first dorsal fin base, second dorsal fin height 0.6 times first dorsal fin height.

Anal fin (Fig. 1) triangular, slightly high, apically narrow, and not falcate. Anal fin anterior margin nearly straight, apex rounded, free rear tip acutely pointed, and posterior margin straight. Anal fin base 0.8 times interdorsal space and 1.3 times dorsal-caudal distance. Anal fin anterior margin 1.5 times its posterior margin and 1.0 times its base. Anal fin height 0.5 times its base and 1.4 times caudal peduncle height. Anal fin larger than second dorsal fin, base 1.4 times second dorsal fin base, and anal fin height 1.0 times second dorsal fin height. 
Caudal fin (Fig. 1) narrow-lobed and asymmetrical, with developed terminal lobe. All caudal fin margins nearly straight, all tips rounded. Dorsal caudal margin without lateral undulations and crest of denticles. Dorsal caudal margin 0.3 times precaudal length and 2.1 times preventral caudal margin. Subterminal margin 0.6 times terminal margin.

Total vertebral counts 112 (109-115 in paratypes), monospondylous vertebral counts 34 (34-36), and precaudal vertebral counts 77 (71-78).

Intestinal valve type conicospiral, with 8 turns (7-8 turns in paratypes).

Dermal denticles (Fig. 6) with flat, elongated, teardrop-shaped crowns. Crown with a strong medial ridge extending its entire length onto a long principal cusp. Dermal denticles above pectoral fin present three ridges, with medial ridge less prominent than in denticles of other regions. Denticles below dorsal fins are longer and present a prominent medial ridge, extending to distal tip of cusplet. In all regions, lateral ridges do not extend beyond intersection between principal cusp and cusplets, and are less prominent. Lateral cusps of denticles well developed above pectoral fin, but not well developed below dorsal fins.

Color pattern (Figs. 1, 4A, B) with dark brown saddles on a brown background. Light spots present on dorsolateral surfaces and fins, spiracle-sized or larger. Dark spots present on lateral body and fins, smaller than spiracles and light spots. Outlines of dark spots not distinct. Light spots on saddles slightly smaller than on body, with spots largest on fins and smallest on saddles. Saddles darker than background, with pectoral saddles extending diagonally forward. Ventral region white to cream, turning dark anterior of the nostrils.

\section{Distribution.}

This species was recorded from the waters around the Izu Islands, Japan (Fig. 7). All specimens were captured by longline fishing for Splendid alfonsino, at depths of ca. 100-200 m around Mikurajima Island, ca. 200-400 m around Hachijojima Island, and ca. 500-600 m around Torishima Island.

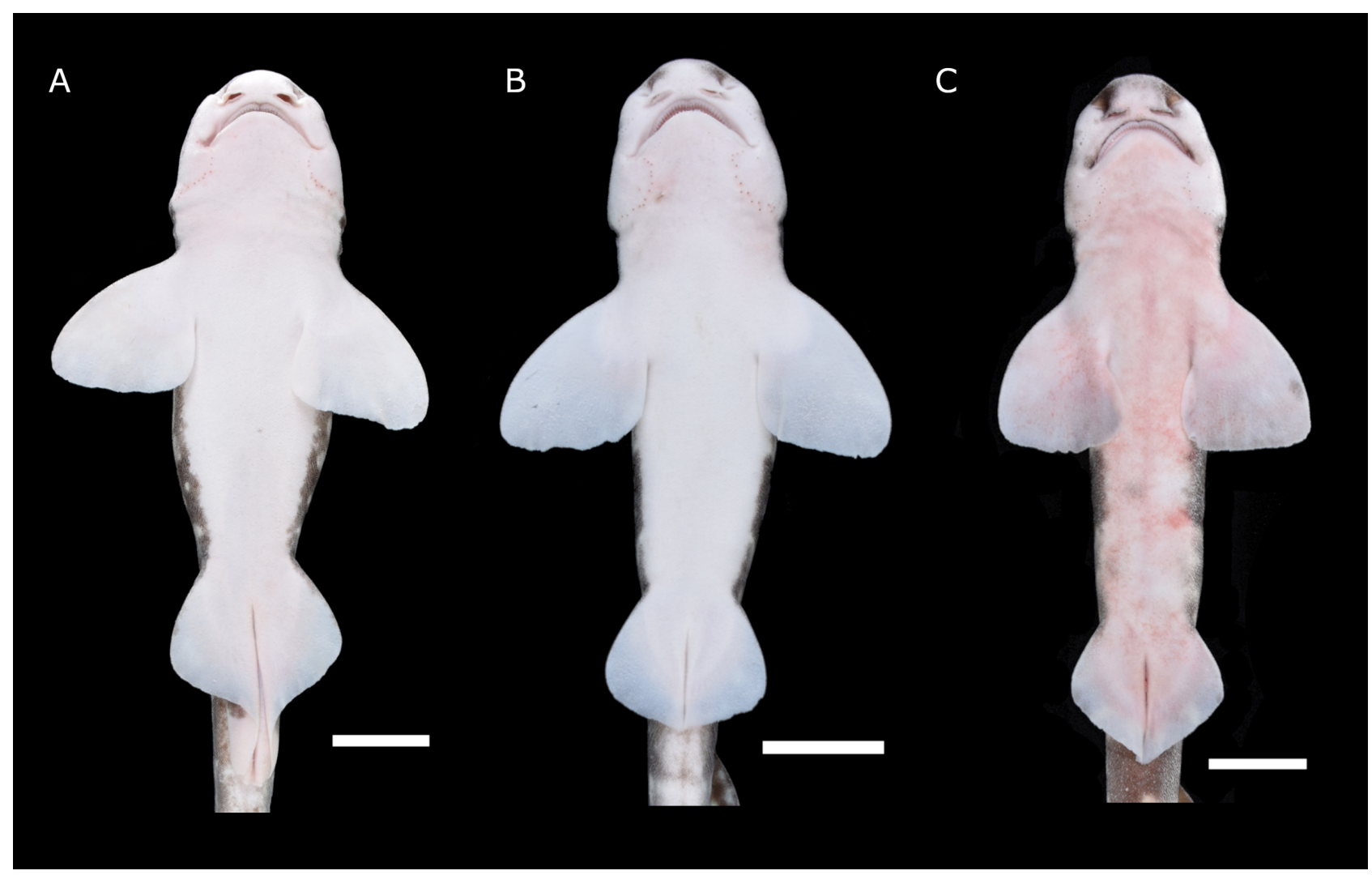

FIG. 4. Ventral view of (A, B) Scyliorhinus hachijoensis and (C) S. torazame specimens. (A) NSMT-P 135960, male, 370 mm TL (off the east coast of Hachijojima Island). (B) NSMT-P 135961, female, $322 \mathrm{~mm}$ TL (off the east coast of Hachijojima Island). (C) NSMT-P 136479, female, 407 mm TL (35¹0.04'N 139³0.08'E, Misaki, Miura, Kanagawa, Japan). Scale bar $=30$ $\mathrm{mm}$. 


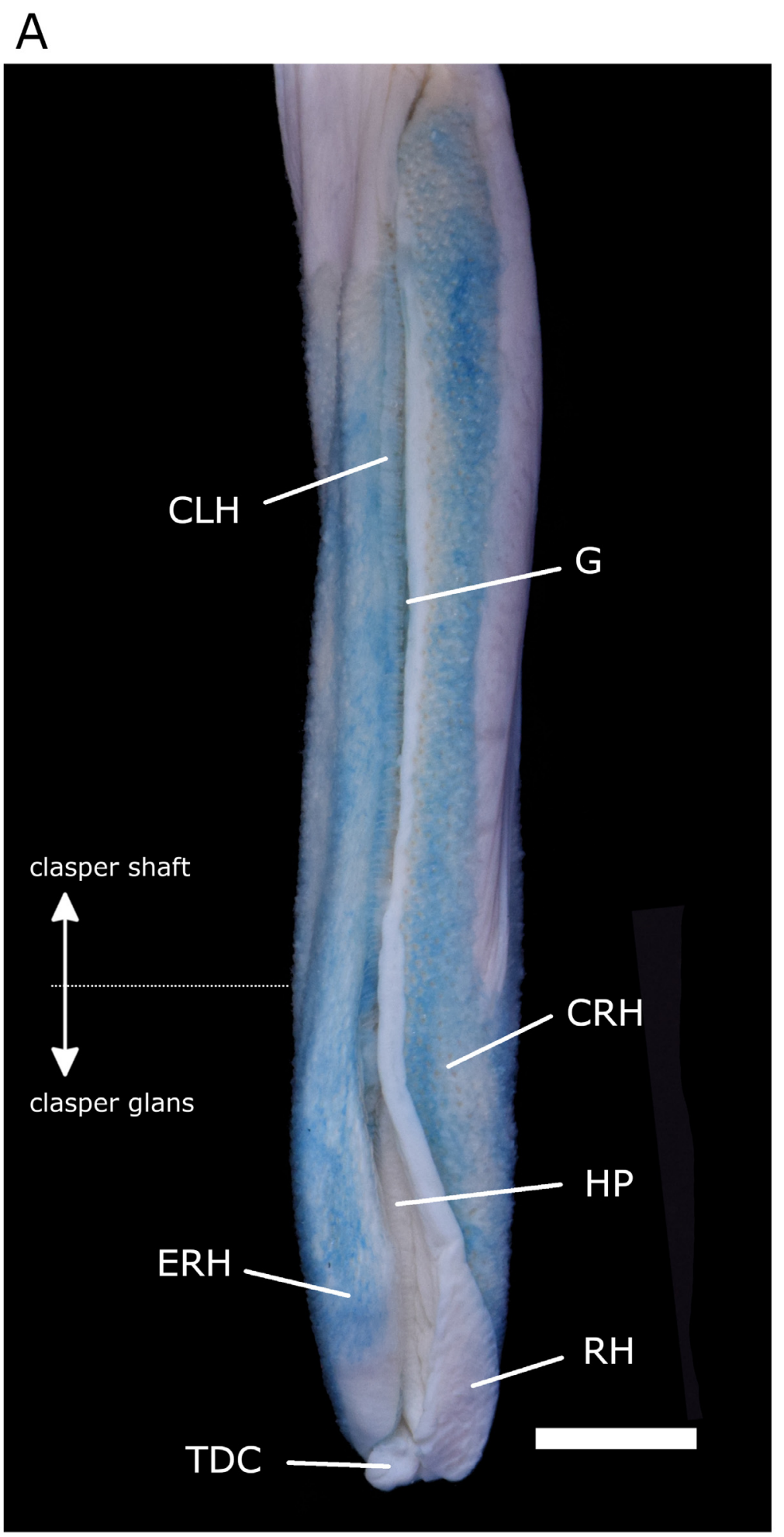

B
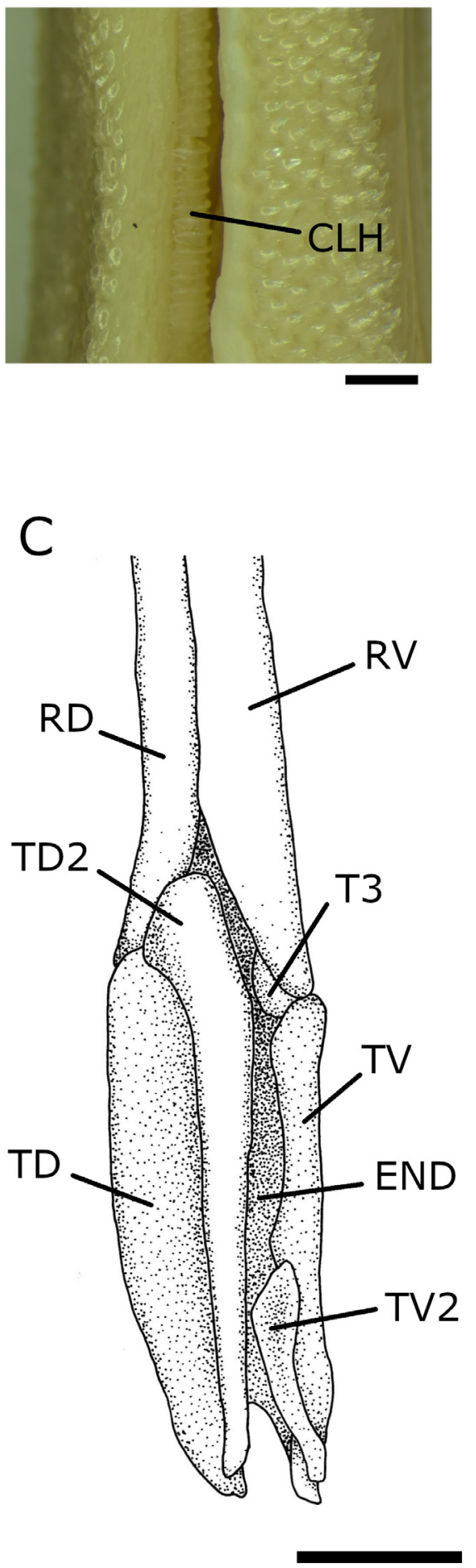

FIG. 5. Clasper of Scyliorhinus hachijoensis. All panels show dorsal views of specimen NSMT-P 135965, 419 mm TL (off the east coast of Torishima Island). (A) External morphology, scale bar $=5 \mathrm{~mm}$. (B) Close-up of clasper hooks, scale bar $=1 \mathrm{~mm}$. (C) Skeleton, scale bar $=5 \mathrm{~mm}$. AP, apopyle; CLH, clasper hooks; CRH, cover rhipidion; END, endstyle; ERH, exorhipidion; G, clasper groove; HP, hypopyle; RD, dorsal marginal cartilage; RH, rhipidion; RV, ventral marginal cartilage; T3, accessory terminal cartilage; TD, dorsal terminal cartilage; TD2, dorsal terminal 2 cartilage; TDC, terminal dermal cover; TV, ventral terminal cartilage; TV2, ventral terminal 2 cartilage. 

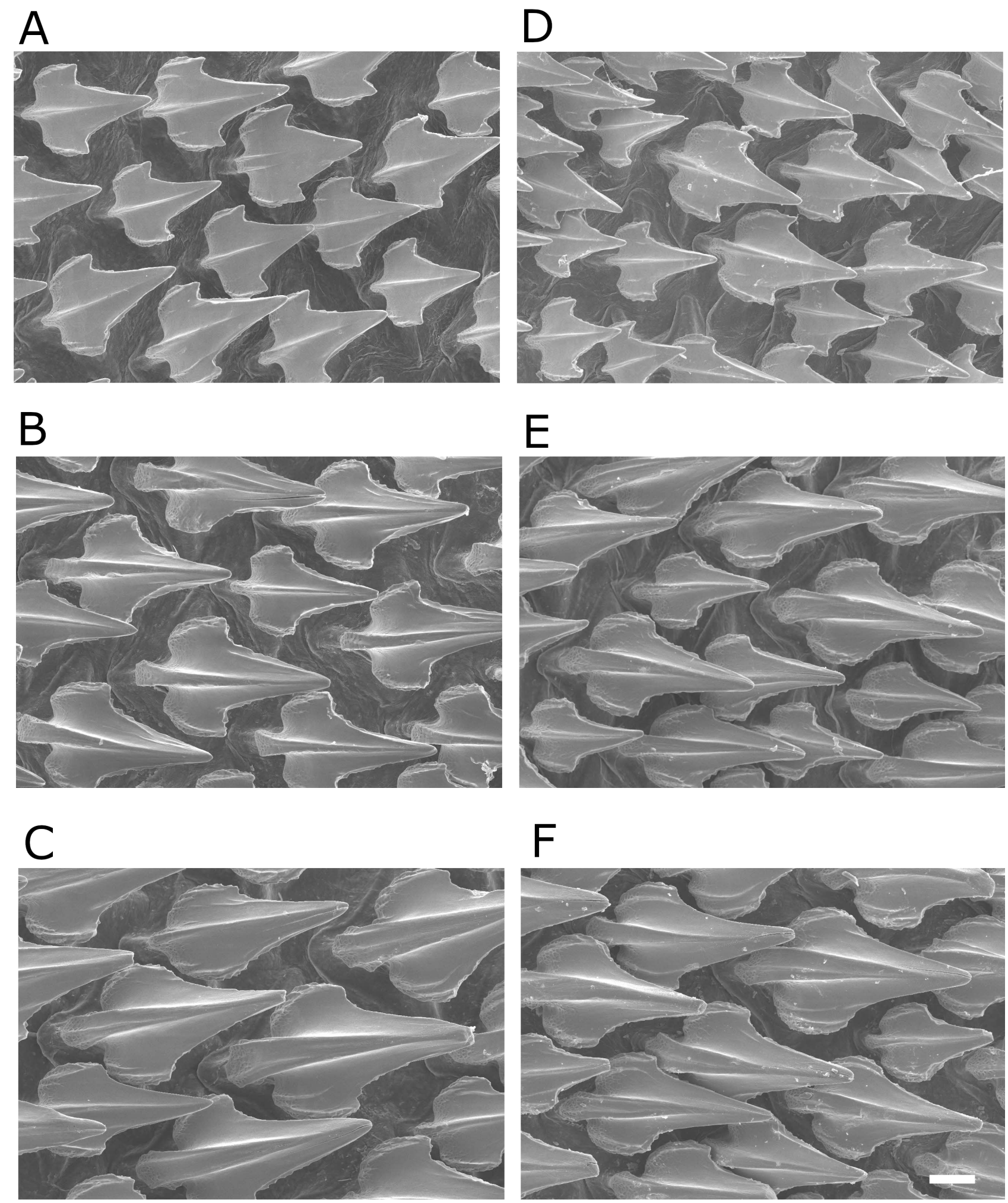

FIG. 6. Dermal denticles of Scyliorhinus hachijoensis. (A-C) NSMT-P 135965, male, $419 \mathrm{~mm}$ TL (off the east coast of Torishima Island). (D-E) NSMT-P 135962, female, $348 \mathrm{~mm}$ TL (off the east coast of Hachijojima Island). Panels show denticles located (A, D) above the pectoral fin base, (B, E) below the first dorsal fin base, and (C, F) below the second dorsal fin base. Scale bar $=200 \mu \mathrm{m}$. 


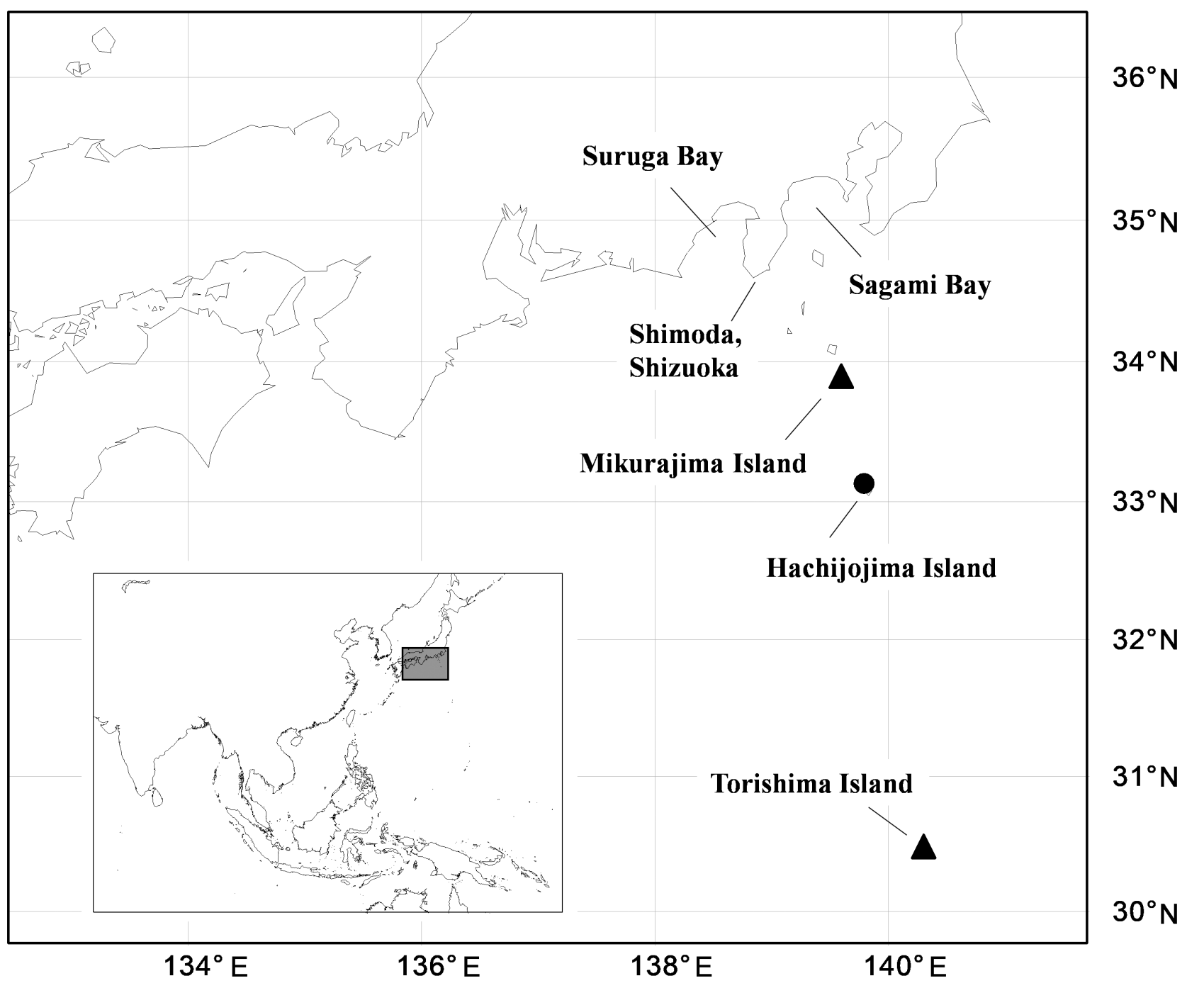

FIG. 7. Distribution of Scyliorhinus hachijoensis. Circle and triangles show catch locations of holotype and paratypes, respectively.
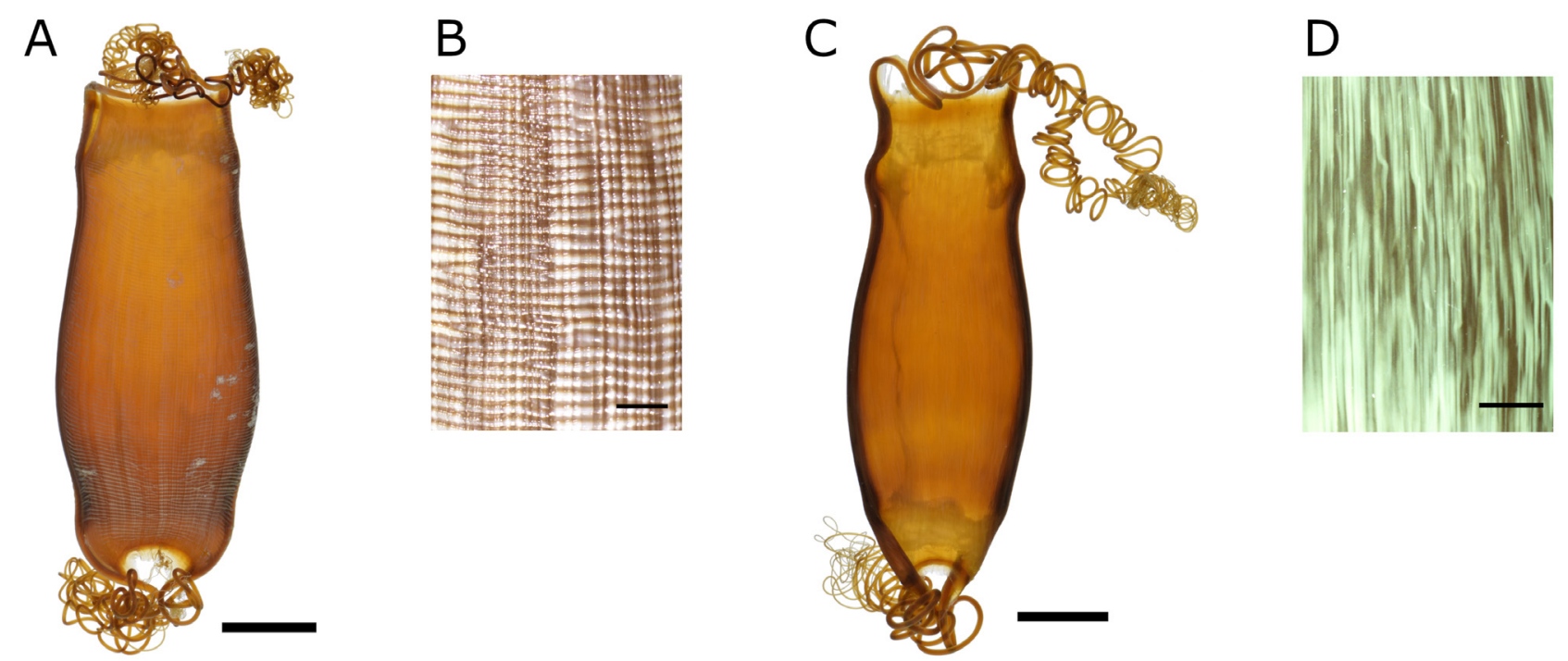

FIG. 8. Egg cases of (A, B) Scyliorhinus hachijoensis and (C, D) S. torazame. (A, C) Dorsal view, scale bar = $10 \mathrm{~mm}$. (B, D) Close-up of surface. Scale bar $=2 \mathrm{~mm}$. 


\section{Biological data.}

Males ranged in size from 294 to $416 \mathrm{~mm}$ TL $(n=6)$ and females from 301 to $386 \mathrm{~mm}$ TL ( $n=22)$. Size at first maturity is not precisely defined, but males had well-developed claspers at $346 \mathrm{~mm}$ TL and females had egg cases at $342 \mathrm{~mm}$. Reproduction is by single oviparity, one egg case for each oviduct. Egg cases are amber in color, with tendrils and surface irregularities that resemble wrinkles (Fig. 8). Maximum case length without tendrils (ML) was 44.6-56.25 mm, and maximum case width was 19.45-22.5 mm (33.4-42.1\%ML) ( $n=7)$.

Stomachs were found to contain small crustaceans, small bony fishes, and polychaets.

\section{Genetic data.}

A total of 2164 base pairs (bp) from three mitochondrial DNA regions were examined: $487 \mathrm{bp}$ for the $16 \mathrm{~S}$ region, $664 \mathrm{bp}$ for COI, and $1013 \mathrm{bp}$ for Cytb. The monophyly of S. hachijoensis was strongly supported by molecular phylogenetic analysis (Fig. 9). The genetic distances between S. hachijoensis and S. torazame were 0.3702 (16S), 0.0295 (COI) and 0.0403 (Cytb), respectively.

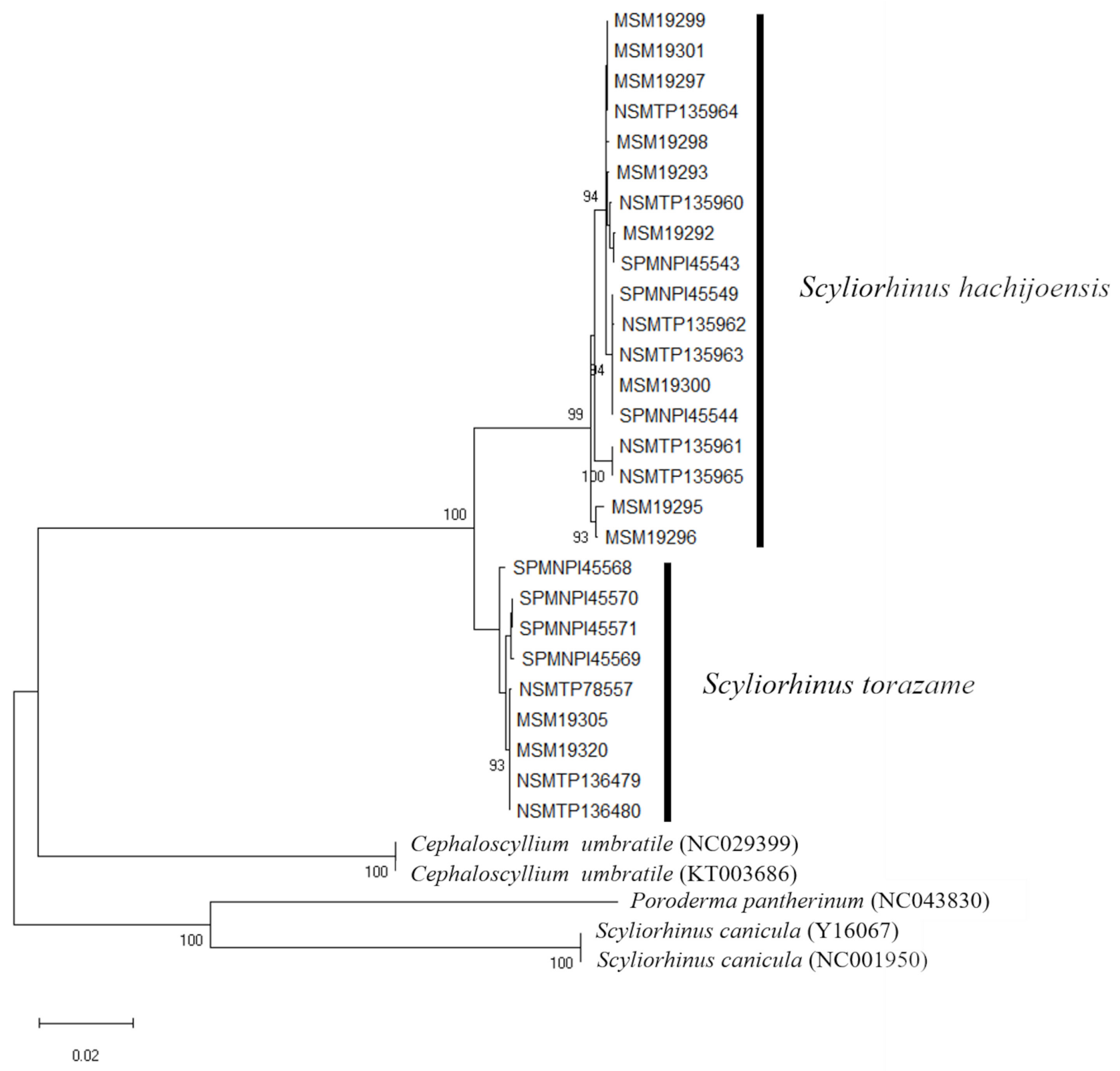

FIG. 9. Maximum likelihood tree of $16 \mathrm{~S}$ rRNA, cytochrome c oxidase subunit I, and cytochrome $b$ sequences from specimens of Scyliorhinus hachijoensis and S. torazame from Japan. Numbers at the nodes are bootstrap values (only values $>90$ are shown). Sample names of Scyliorhinus hachijoensis and S. torazame correspond to museum-deposited numbers (see APPENDIX 1). 


\section{Etymology.}

The species name "hachijoensis" refers to the species' main collection area, Hachijojima Island. The English name is derived from "Cinderella", because the dark spots on the body surface are similar to black ashes "cinder". The Japanese name "Fukami" means "deep sea".

\section{Remarks.}

Clasper hooks are a unique character observed in S. torazame (Soares \& de Carvalho 2019, 2020), and the same character was also observed in S. hachijoensis.

Both male and female $S$. hachijoensis can be easily distinguished from eleven other Scyliorhinus species by color pattern: S. cervigoni Maurin \& Bonnet, 1970, S. garmani (Fowler, 1934), S. meadi Springer, 1966 and $S$. retifer (Garman, 1881) have no light spots, S. capensis (Müller \& Henle, 1838) has light yellow to golden spots, and S. torrei Howell-Rivero, 1936 has beige to cream spots. Scyliorhinus canicula, S. cabofriensis Soares, Gomes \& de Carvalho, 2016, S. cervigoni, S. garmani, S. haeckelii (Miranda Ribeiro, 1907) and S. stellaris (Linnaeus, 1758) have conspicuous dark spots, and S. boa (Goobe \& Bean, 1896) has various dark spots (Springer 1979; Ebert et al. 2013a; Soares \& de Carvalho 2019). Scyliorhinus comoroensis Compagno, 1988 and S. duhamelii (Garman, 1913) (as well as S. canicula, S. cervigoni, S. garmani and S. stellaris, which are also listed above) can be distinguished from S. hachijoensis because the nasal flaps reach or cover the upper lip (Springer 1979; Ebert et al. 2013a; Soares \& de Carvalho 2019). Scyliorhinus hesperius Springer, 1966 and S. ugoi Soares, Gomes \& Gadig, 2015 are very similar to S. hachijoensis in color pattern, but differ in the number of monospondylous vertebrae and size of maturity. The number of monospondylous vertebrae is 39-42 in S. hesperius and 38-39 in S. ugoi. Adult males mature at least $420 \mathrm{~mm}$ TL in $S$. hesperius, and by at least $450 \mathrm{~mm}$ TL and $470 \mathrm{~mm}$ TL in male and female specimens of $S$. ugoi, respectively. By contrast, the number of monospondylous vertebrae in S. hachijoensis is 34-36 and the size at maturity is less than $350 \mathrm{~mm}$ TL. In addition, $S$. ugoi has saddles with antero- and posteromedial projections (Springer 1979; Ebert et al. 2013a; Soares et al. 2015; Soares \& de Carvalho 2019).

Scyliorhinus torazame is similar in external characters to $S$. hachijoensis, but differs in fin height and fin angle. In $S$. torazame, anal fin height is less than $4 \% \mathrm{TL}$ and approximately equal to caudal peduncle height (as compared to more than $4 \% \mathrm{TL}$ and higher than the caudal peduncle in $S$. hachijoensis) (Table 1), the pectoral fin angle in adults is $70^{\circ}-96^{\circ}$ and the pelvic fin angle in adult females is $40^{\circ}-65^{\circ}$ (as compared to a pectoral fin angle of $90^{\circ}-102^{\circ}$ and pelvic fin angle of $63^{\circ}-75^{\circ}$ in $S$. hachijoensis), the pelvic fin apex is located in the middle of the fin (vs. apex obviously behind the middle of the fin in S. hachijoensis) (Figs. 1, 4), and the dermal denticles present five ridges (vs. three ridges in S. hachijoensis). Also, S. torazame has no dark spots. Finally, whereas the egg case surface is irregularities that resemble wrinkles in S. hachijoensis, the egg case is smooth in S. torazame (Fig. 8).

Previously, S. torazame had not been reported from any waters farther south than Taiwan. Also, reports of this species from Okinawa have been rare (Springer 1979; Carpenter 1998; Ebert et al. 2013a; Soares \& de Carvalho 2019). In this study, we identified numerous specimens from the Ryukyu Islands (specifically, the islands of Okinawa and Haterumajima) and Taiwan, including the first confirmed specimen from the South China Sea, thus expanding the known distribution of S. torazame (see APPENDIX 1).

\section{Acknowledgements}

We acknowledge the following museum staff for their assistance in accessing materials from their collections: G. Shinohara and M. Nakae (NSMT), H. Tashiro (HUMZ), Y. Kai (FAKU), H. Senou (KPM), K. Hoshino (SNFR), H. Endo (BSKU), and H-C. Ho (NMMB).

Some specimens examined in this study were captured as part of the "Project to promote stock surveys and assessments of fisheries resources of waters around Japan" promoted by the Fisheries Agency, Japan. The following people assisted with the collection of fresh material during this study: Y. Narimatsu, R. Misawa, and the captain and crew of the Wakatakamaru (Tohoku National Fisheries Research Institute); T. Ishibashi, M. Ishibashi, and N. Shimomura (Shimonoseki Marine Science Museum); K. Kofuji (Ibaraki Prefectural Oarai Aquarium); T. Nakai (Keikyu Aburatsubo Marine Park); M. Kojima (Niigata City Aquarium); Y. Otani and the captain and crew of the Tajima (Tajima Fisheries Technology Institute, Hyogo Prefectural Technology Center for Agriculture, Forestry and Fisheries); A. Sasaki (Kagoshima City Aquarium); M. Todate (Takeshima Aquarium); and the captains and crews of 
the Zoeimaru, No. 3 Idoinkyomaru, Koeimaru, Eishinmaru, Chokomaru, No. 88 Takamaru, No. 10 Hukuichimaru and Asashiomaru.

We also thank the following for providing their expert opinions and other invaluable information: T. Isshiki (Kanagawa Prefectural Fisheries Technology Center Sagamiwan Experimental Station); K. Sato and K. Miyamoto (Okinawa Churashima Foundation Research Center); S. Chiba (National Research Institute of Far Seas Fisheries); K. Koeda (Kuroshio Biological Research Institute); N. Nakayama, T. Horie and H. Takeshima (School of Marine Science and Technology, Tokai University); K. Fujiwara, N. Tsuzuki, and T. Okano (Shimoda Floating Aquarium); S. Tomiyama (Tokai University Marine Science Museum); K. Shibukawa (Museum of Natural and Environmental History).

\section{References}

Bustamante, C., Kyne, P. M. \& Bennett, M.B. (2013) Comparative morphology of the egg cases of Asymbolus analis, Asymbolus rubiginosus and Figaro boardmani (Carcharhiniformes: Scyliorhinidae) from southern Queensland, Australia. Journal of Fish Biology, 83, 133-143. https://doi.org/10.1111/jfb.12155

Carpenter, K.E. (1998) FAO species identification guide for fishery purposes. The living marine resources of the Western Central Pacific. Vol. 2. Cephalopods, crustaceans, holothurians and sharks. FAO, Rome, 710 pp. [pp. 687-1396]

Compagno, L.J.V. (1984) FAO species catalogue. Vol. 4. Sharks of the World: An annotated and illustrated catalogue of shark species known to date. Part 2. Carcharhiniformes. FAO Fisheries Synopsis, 125 (4), 251-655.

Compagno, L.J.V. (1988) Sharks of the Order Carcharhiniformes. Princeton University Press, Princeton, New Jersey, 542 pp.

Compagno, L.J.V. (2001) Shark of the world. An annotated and illustrated catalogue of sharks known to date. Vol. 2. Bullhead, mackerel and carpet sharks (Heterodontiformes, Lamniformes and Orectolobiformes). FAO species catalogue for fishery purposes, Rome, $269 \mathrm{pp}$.

Diepenbroek, M., Grobe, H. \& Sieger, R. (2000) PanMap. Available from: http://www.pangaea.de/Software/PanMap (accessed 29 December 2021)

Dyldin, Y.V. (2015) Annotated checklist of the sharks, batoids and chimaeras (Chondrichthyes: Elasmobranchii, Holocephali) from waters of Russia and adjacent areas. Publications of the Seto Marine Biological Laboratory, 43, 40-91. https://doi.org/10.5134/197957

Ebert, D.A., Fowler, S. \& Compagno, L. (2013a) Sharks of the World: A Fully Illustrated Guide. Wild Nature Press, Plymouth, $528 \mathrm{pp}$.

Ebert, D.A., White, W.T., Ho, H.-C., Last, P.R., Nakaya, K., Séret, B., Straube, N., Naylor, G.J.P. \& de Carvalho, M.R. (2013b) An annotated checklist of the chondrichthyans of Taiwan. Zootaxa, 3752 (1), 279-386. https://doi.org/10.11646/zootaxa.3752.1.17

Fricke, R. \& Eschmeyer, W.E. (2020) A guide to fish collections in the Catalog of Fishes. Online Version 7 December 2020. Available from: http://researcharchive.calacademy.org/research/ichthyology/catalog/collections.asp (Accessed 7 Dec. 2020)

Hagiwara, S. (1993) Keeping and Reproduction of Chondrichthyans in Captivity at Shimoda Floating Aquarium. Report of Japanese Society for Elasmobranch Studies, 30, 1-18.

Herman, J., Hovestadt-Euler, M. \& Hovestadt, D.C. (1993) Contributions to the study of the comparative morphology of teeth and other relevant ichthyodorulites in living supraspecific taxa of Chondrichthyan fishes. Part A: Selachii. No. 2b: Order: Carcharhiniformes_Family: Scyliorhinidae. Bulletin de l'institut Royal des Sciences Naturalles de Belgique, Biology, 60, 181-230.

Ivanova, N.V., Zemlak, T.S., Hanner, R.H. \& Hebert, P.D.N. (2007) Universal primer cocktails for fish DNA barcoding. Molecular Ecology Notes, 7, 544-548. https://doi.org/10.1111/j.1471-8286.2007.01748.x

Kim, B.J., Kim, I.S., Nakaya, K., Yabe, M., Cho, Y. \& Imamura, H. (2009) Checklist of the Fishes from Jeju Island, Korea. Bulletin of the Faculty of Fisheries Hokkaido University, 59 (1), 7-36.

Kumar, S., Stecher, G., Li, M., Knyaz, C., \& Tamura, K. (2018) MEGA X: Molecular Evolutionary Genetics Analysis across computing platforms. Molecular Biology and Evolution, 35, 1547-1549. https://doi.org/10.1093/molbev/msy096

Nakaya, K. (1975) Taxonomy, Comparative Anatomy and Phylogeny of Japanese Catsharks, Scyliorhinidae. Memories of the Faculty of Fisheries, Hokkaido University, 23 (1), 1-94.

Pietschmann, V. (1906) Ichthyologische Ergebnisse einer Reisen ach Island, an die atlantische Küste von Marokko und in die westliche Hälfte des Mittelmeeres. Annalen des Naturhistorischen Museums in Wien, 21, 72-148.

Pietschmann, V. (1908) Zwei neue japanische Haifische. Anzeiger der Akademie der Wissenschaften in Wien, 45 (10), $132-$ 135.

Shirai, S., Hagiwara, S. \& Nakaya, K. (1992) Scyliorhinus tokubee sp. nov. from Izu Peninsula, Southern Japan (Scyliorhinidae, Elasmobranchii). Japanese Journal of Ichthyology, 39 (1), 9-16. https://doi.org/10.11369/jji1950.39.9 
Soares, K.D.A. (2020) Comparative anatomy of the clasper of catsharks and its phylogenetic implications (Chondrichthyes: Carcharhiniformes: Scyliorhinidae). Journal of Morphology, 281, 591-607.

https://doi.org/10.1002/jmor.21123

Soares, K.D.A. \& de Carvalho, M.R. (2019) The catshark genus Scyliorhinus (Chondrichthyes: Carcharhiniformes: Scyliorhinidae): taxonomy, morphology and distribution. Zootaxa, 4601 (1), 1-147. https://doi.org/10.11646/zootaxa.4601.1.1

Soares, K.D.A. \& de Carvalho, M.R. (2020) Phylogenetic relationships of catshark species of the genus Scyliorhinus (Chondrichthyes, Carcharhiniformes, Scyliorhinidae) based on comparative morphology. Zoosystematics and Evolution, 96 (2), 345-395.

https://doi.org/10.3897/zse.96.52420

Soares, K.D.A., Gadig, O.F.B. \& Gomes, U.L. (2015) Scyliorhinus ugoi, a new species of catshark from Brazil (Chondrichthyes: Carcharhiniformes: Scyliorhinidae). Zootaxa, 3937 (2), 347-361. https://doi.org/10.11646/zootaxa.3937.2.6

Springer, S. (1979) A revision of the catsharks, family Scyliorhinidae. NOAA technical report NMFS circular, $422,1-97$. https://doi.org/10.5962/bhl.title.63029

Springer, V.G. (1964) A revision of the Carcharhinid shark genera Scoliodon, Loxodon, and Rhizoprionodon. Proceedings of the United States National Museum, 115 (3493), 559-632. https://doi.org/10.5479/si.00963801.115-3493.559

Tamura, K. \& Nei, M. (1993) Estimation of the number of nucleotide substitutions in the control region of mitochondrial DNA in humans and chimpanzees. Molecular Biology and Evolution, 10, 512-526.

Weigmann, S. (2016) Annotated checklist of the living sharks, batoids and chimaeras (Chondrichthyes) of the world, with a focus on biogeographical diversity. Journal of Fish Biology, 88 (3), 837-1037.

https://doi.org/10.1111/jfb.12874

\section{APPENDIX 1. Comparative materials.}

Scyliorhinus torazame

HUMZ 107358, male, $410 \mathrm{~mm}$ TL (Shimoda, Shizuoka, Japan-holotype of S. tokubee); HUMZ 113574, female, $391 \mathrm{~mm}$ TL (Shimoda, Shizuoka, Japan - paratype of S. tokubee); HUMZ 113578, female, $378 \mathrm{~mm}$ TL (Shimoda, Shizuoka, Japan - paratype of S. tokubee); HUMZ 117472, female, 378 mm TL (Shimoda, Shizuoka, Japan-paratype of S. tokubee); HUMZ 117496, male, $384 \mathrm{~mm}$ TL (Shimoda, Shizuoka, Japan — paratype of $S$. tokubee); NSMT-P 34976, female, $381 \mathrm{~mm}$ TL (Shirahama, Shizuoka, Japan, $100 \mathrm{~m}$ depth — paratype of S. tokubee); ASIZP 57958, male, $194 \mathrm{~mm}$ TL, male, $166 \mathrm{~mm}$ TL (243․ ${ }^{\circ} 1^{\prime} \mathrm{N} 121^{\circ} 52.01^{\prime} \mathrm{E}$, Nanfangao, Yilan, Taiwan); BSKU 115160, male, 240 mm TL (Hachinohe, Aomori, Japan); BSKU 115161, male, 155 mm TL (Hachinohe, Aomori, Japan); FAKU no number, male, $435 \mathrm{~mm}$ TL (Haterumajima Island, Japan); FAKU 5727, female, $403 \mathrm{~mm}$ TL (Haterumajima Island, Japan); FAKU 7569, female, $382 \mathrm{~mm}$ TL (Haterumajima Island, Japan); FAKU 13723, male, 404 mm TL (Haterumajima Island, Japan); FAKU 13914, female, 405 mm TL (Haterumajima Island, Japan); FAKU 31905, female, $372 \mathrm{~mm}$ TL (Haterumajima Island, Japan); FAKU 75430, male, $423 \mathrm{~mm}$ TL (Tachibana Bay, Nagasaki, Japan); FAKU 75431, male, 413 mm TL (Tachibana Bay, Nagasaki, Japan); FAKU 131312, male, 438 mm TL (Maizuru, Kyoto, Japan); FAKU 131729, male, 442 mm TL (Ine, Kyoto, Japan); FAKU 131730, female,

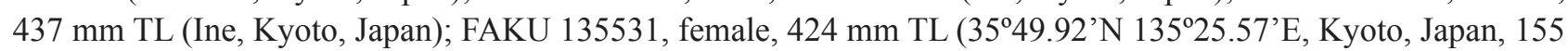
m depth); FAKU 135817, female, 450 mm TL (Miyazu, Kyoto, Japan); FAKU 137000, female, 428 mm TL (western Wakasa Bay, Kyoto, Japan); KPM-NI 40163, male, 462 mm TL (Moroiso, Misaki, Kanagawa, Japan); KPM-NI 40164, female, 465 mm TL (Moroiso, Misaki, Kanagawa, Japan); KPM-NI 40165, female, 389 mm TL (Moroiso, Misaki, Kanagawa, Japan); KPM-NI 23080, female, 429 mm TL (Nagai, Yokosuka, Kanagawa, Japan); KPM-NI 47684, female, 385 mm TL (Numazu, Shizuoka, Japan); HUMZ 39414, female, $344 \mathrm{~mm}$ TL (30³6’ N 13003'E, Yakushima Island, Japan); HUMZ 49642, male, 171 mm TL (Mitani fish market, Aichi, Japan); HUMZ 49643, male, 143 mm TL (Mitani fish market, Aichi, Japan); HUMZ 49644, sex unknow, 130 mm TL (Mitani fish market, Aichi, Japan); HUMZ 111033, male, 341 mm TL (Shimoda, Shizuoka, Japan); HUMZ 111034, male, 367 mm TL (Shimoda, Shizuoka, Japan); HUMZ 189927, female, 392 mm TL (Hakodate Bay, Hokkaido, Japan); HUMZ 189934, female, 463 mm TL (Hakodate Bay, Hokkaido, Japan); HUMZ 197854, female, 384 mm TL (Wakimoto

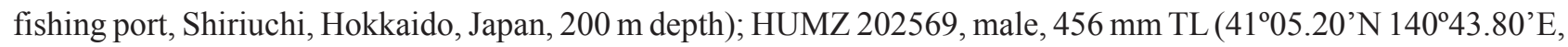
Mutsu Bay, Kaizaki, Aomori, Japan, $62 \mathrm{~m}$ depth); HUMZ 202570, male, $445 \mathrm{~mm}$ TL (41 $05.20^{\prime} \mathrm{N} 140^{\circ} 43.80^{\prime} \mathrm{E}$, Mutsu Bay, Kaizaki, Aomori, Japan, 62 m depth); HUMZ 210796, female, 408 mm TL (Nanaehama, Hokkaido, Japan); HUMZ 230732, male, 388 mm TL (hakodate port, Nanaehama, Hokkaido, Japan); MSM-19-302, female, 
417 mm TL (Ose, Ibaraki, Japan); MSM-19-303, female, 419 mm TL (Ose, Ibaraki, Japan); MSM-19-304, male, 455 mm TL (Ose, Ibaraki, Japan); MSM-19-305, male, 456 mm TL (Ose, Ibaraki, Japan); MSM-19-306, female, 368 mm TL (36 $59.93^{\prime} \mathrm{N} 141^{\circ} 17.56$ 'E, Hitachi, Ibaraki, Japan, $150 \mathrm{~m}$ depth); MSM-19-307, female, $390 \mathrm{~mm}$ TL (36 $59.93^{\prime} \mathrm{N} 141^{\circ} 17.56^{\prime} \mathrm{E}$, Hitachi, Ibaraki, Japan, $150 \mathrm{~m}$ depth); MSM-19-308, female, $412 \mathrm{~mm}$ TL (36 $59.93^{\circ} \mathrm{N}$ $141^{\circ} 17.56^{\prime} \mathrm{E}$, Hitachi, Ibaraki, Japan, $150 \mathrm{~m}$ depth); MSM-19-309, male, $255 \mathrm{~mm}$ TL (36 $30.32^{\prime} \mathrm{N} 140^{\circ} 57.36^{\prime} \mathrm{E}$, Mito, Ibaraki, Japan, $150 \mathrm{~m}$ depth); MSM-19-310, female, $233 \mathrm{~mm}$ TL (36 30.32'N 140'57.36'E, Mito, Ibaraki, Japan, 150 m depth); MSM-19-311, female, 207 mm TL (36 30.32’'N 140 $57.36^{\circ} \mathrm{E}$, Mito, Ibaraki, Japan, $150 \mathrm{~m}$ depth); MSM-19-312, female, 163 mm TL (36 30.32'N 14057.36’E, Mito, Ibaraki, Japan, 150 m depth); MSM-19313, male, $138 \mathrm{~mm}$ TL (36 30.32'N 14057.36'E, Mito, Ibaraki, Japan, $150 \mathrm{~m}$ depth); MSM-19-314, female, 129 mm TL ( $36^{\circ} 30.32^{\prime} \mathrm{N} 140^{\circ} 57.36^{\prime} \mathrm{E}$, Mito, Ibaraki, Japan, $150 \mathrm{~m}$ depth); MSM-19-315, male, $95 \mathrm{~mm}$ TL (36 $30.32^{\prime} \mathrm{N}$

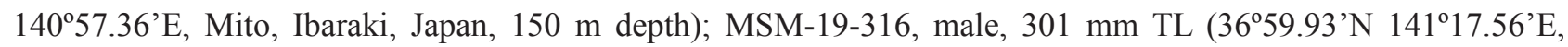
Hitachi, Ibaraki, Japan, 150 m depth); MSM-19-317, female, 240 mm TL (36 $59.93^{\prime} \mathrm{N} 141^{\circ} 17.56$ 'E, Hitachi, Ibaraki, Japan, 150 m depth); MSM-19-318, female, 213 mm TL (36 $59.93^{\prime} \mathrm{N} 141^{\circ} 17.56^{\prime} \mathrm{E}$, Hitachi, Ibaraki, Japan, $150 \mathrm{~m}$ depth); MSM-19-319, female, 184 mm TL (36 $59.93^{\prime} \mathrm{N} 141^{\circ} 17.56^{\prime} \mathrm{E}$, Hitachi, Ibaraki, Japan, $150 \mathrm{~m}$ depth); MSM19-320, male, 256 mm TL (36 $59.93^{\prime} \mathrm{N} 141^{\circ} 17.56^{\prime} \mathrm{E}$, Hitachi, Ibaraki, Japan, $150 \mathrm{~m}$ depth); MSM-19-321, female, $290 \mathrm{~mm}$ TL (36 59.93'N 141 ${ }^{\circ} 17.56^{\prime} \mathrm{E}$, Hitachi, Ibaraki, Japan, $150 \mathrm{~m}$ depth); NMMB-P 033383, male, $341 \mathrm{~mm}$ TL $\left(24^{\circ} 21.60^{\prime} \mathrm{N} 122^{\circ} 24.00^{\prime} \mathrm{E}\right.$, Yilan, Taiwan, $460 \mathrm{~m}$ depth); NSMT-P 5952, male, $87 \mathrm{~mm}$ TL (Mamezu, Tsushima, Nagasaki, Japan, $100 \mathrm{~m}$ depth); NSMT-P 50323, female, $341 \mathrm{~mm}$ TL (southeast off Tsushima, Nagasaki, Japan); NSMT-P 50333, male, $368 \mathrm{~mm}$ TL (off the southeast coast of Tsushima, Nagasaki, Japan); NSMT-P 50625, male, $395 \mathrm{~mm}$ TL (off the southeast coast of Tsushima, Nagasaki, Japan); NSMT-P 50626, female, $280 \mathrm{~mm}$ TL (off the southeast coast of Tsushima, Nagasaki, Japan); NSMT-P 52632, male, 416 mm TL (Kanaya, Chiba, Japan); NSMTP 61264, female, $384 \mathrm{~mm}$ TL (36 $29.10^{\prime} \mathrm{N}$ 140 58.40'E, Ibaraki, Japan); NSMT-P 63286, male, $416 \mathrm{~mm}$ TL

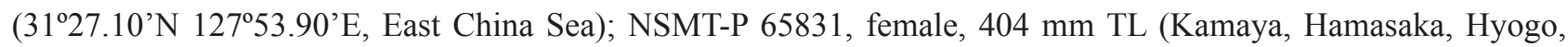
Japan); NSMT-P 65836, female, 209 mm TL (Kamaya, Hamasaka, Hyogo, Japan, 250-270 m depth); NSMT-P 66232, male, $238 \mathrm{~mm}$ TL (Tateyama, Chiba, Japan, 200-300 m depth); NSMT-P 69076, male, $221 \mathrm{~mm}$ TL (31 $21.31^{\prime} \mathrm{N} 128^{\circ} 19.12^{\prime} \mathrm{E}$, East China Sea, $260 \mathrm{~m}$ depth); NSMT-P 74816, male, $400 \mathrm{~mm}$ TL (Hiragata, Hukushima, Japan); NSMT-P 74879, female, 426 mm TL, female, 440 mm TL (Ippongi, Aomori, Japan); NSMT-P 78557, male, $440 \mathrm{~mm}$ TL (36 $31.30^{\circ} \mathrm{N} 140^{\circ} 58.20^{\prime} \mathrm{E}$, Ibaraki, Japan); NSMT-P 79500, female, $385 \mathrm{~mm}$ TL (36 $59.60^{\circ} \mathrm{N} 141^{\circ} 17.40^{\prime} \mathrm{E}$, Ibaraki, Japan); NSMT-P 96552, male, $348 \mathrm{~mm}$ TL (off the southeast coast of Tsushima, Nagasaki, Japan); NSMT-

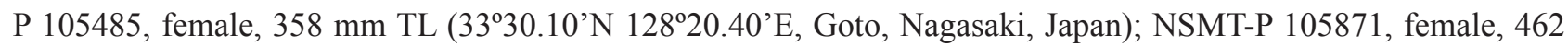
mm TL (Misaki, Kanagawa, Japan); NSMT-P 136479, male, 424 mm TL ( $35^{\circ} 10.04^{\prime} \mathrm{N} 139^{\circ} 30.08^{\prime} \mathrm{E}$, Misaki, Miura, Kanagawa, Japan, $200 \mathrm{~m}$ depth); NSMT-P 136480, female, $407 \mathrm{~mm}$ TL (35'10.04'N 139 $30.08^{\prime} \mathrm{E}$, Misaki, Miura,

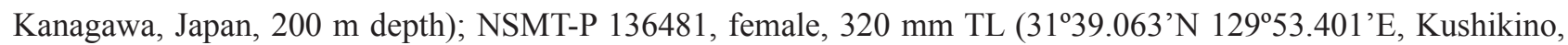
Kagoshima, Japan, $325 \mathrm{~m}$ depth); NSMT-P 136482, female, $327 \mathrm{~mm}$ TL (31 ${ }^{\circ} 39.063^{\prime} \mathrm{N} 129^{\circ} 53.401^{\prime}$ 'E, Kushikino, Kagoshima, Japan, 325 m depth); NSMT-P 136483, female, 385 mm TL (31³9.063'N 12953.401'E, Kushikino, Kagoshima, Japan, 325 m depth); OCF-P 2620, male, 442 mm TL (Okinawa, Japan); OCF-P 3848, male, 400 mm TL (Onna, Okinawa, Japan, $500 \mathrm{~m}$ depth); SNFR 11621, female, $408 \mathrm{~mm}$ TL (South China Sea); SNFR 13396, male, $385 \mathrm{~mm}$ TL (East China Sea); SNFR 14485, female, $374 \mathrm{~mm}$ TL (East China Sea); SNFR 14588, male, 380 mm TL (East China Sea); SNFR 14916, female, 408 mm TL (East China Sea); SNFR 18693, female, 393 mm TL (Niigata, Japan); SNFR 18694, female, 313 mm TL (Niigata, Japan); SNFR 19129, male, $337 \mathrm{~mm}$ TL (East China Sea); SPMN-PI 45552, male, 465 mm TL (Kakutahama, Niigata, Japan, $120 \mathrm{~m}$ depth); SPMN-PI 45553, male, 450 mm TL (Kakutahama, Niigata, Japan, $120 \mathrm{~m}$ depth); SPMN-PI 45554, male, $462 \mathrm{~mm}$ TL (Kakutahama, Niigata, Japan, $120 \mathrm{~m}$ depth); SPMN-PI 45555, female, $413 \mathrm{~mm}$ TL (Kakutahama, Niigata, Japan, $120 \mathrm{~m}$ depth); SPMN-PI 45556, female, $419 \mathrm{~mm}$ TL (Kakutahama, Niigata, Japan, $120 \mathrm{~m}$ depth); SPMN-PI 45557, female, $456 \mathrm{~mm}$ TL (Kakutahama, Niigata, Japan, $120 \mathrm{~m}$ depth); SPMN-PI 45558, male, $375 \mathrm{~mm}$ TL (3440.0'N 130²0.0'E, Japan Sea, Shimonoseki, Yamaguchi, Japan, 126 m depth); SPMN-PI 45559, female, 365 mm TL (31³9.063'N 129 $53.401^{\prime}$ 'E,

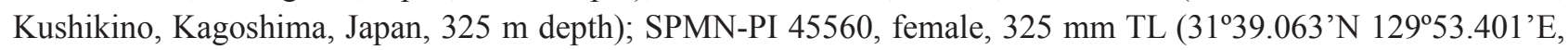

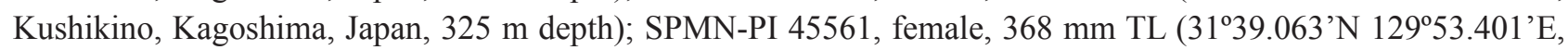
Kushikino, Kagoshima, Japan, 325 m depth); SPMN-PI 45562, male, 375 mm TL (31 $39.063^{\prime} \mathrm{N} 129^{\circ} 53.401^{\prime} \mathrm{E}$, Kushikino, Kagoshima, Japan, $325 \mathrm{~m}$ depth); SPMN-PI 45563, female, $437 \mathrm{~mm}$ TL $\left(32^{\circ} 46.80^{\prime} \mathrm{N} 129^{\circ} 0.60^{\prime} \mathrm{E}\right.$, Narujima Island, Nagasaki, Japan, $92 \mathrm{~m}$ depth); SPMN-PI 45564, female, $410 \mathrm{~mm}$ TL (32 $46.80^{\prime} \mathrm{N} 129^{\circ} 0.60^{\prime} \mathrm{E}$, Narujima Island, Nagasaki, Japan, $92 \mathrm{~m}$ depth); SPMN-PI 45565, male, $362 \mathrm{~mm}$ TL $\left(32^{\circ} 46.80^{\prime} \mathrm{N} 129^{\circ} 0.60^{\prime} \mathrm{E}\right.$, 
Narujima Island, Nagasaki, Japan, $92 \mathrm{~m}$ depth); SPMN-PI 45566, female, $413 \mathrm{~mm}$ TL $\left(32^{\circ} 46.80^{\prime} \mathrm{N} 129^{\circ} 0.60^{\prime} \mathrm{E}\right.$, Narujima Island, Nagasaki, Japan, $92 \mathrm{~m}$ depth); SPMN-PI 45567, female, $382 \mathrm{~mm}$ TL (Oshima Island, Tokyo, Japan); SPMN-PI 45568, female, 370 mm TL (Oshima Island, Tokyo, Japan); SPMN-PI 45569, female, 332 mm TL (Oshima Island, Tokyo, Japan); SPMN-PI 45570, male, 225 mm TL (Oshima Island, Tokyo, Japan); SPMN-PI 45571, male, 203 mm TL (Oshima Island, Tokyo, Japan); URM-P 39137, male, $423 \mathrm{~mm}$ TL (Okinawa, Japan); URM-P 39138, male, 444 mm TL (Okinawa, Japan); URM-P 39139, male, 393 mm TL (Okinawa, Japan); URM-P 39140, male, 437 mm TL (Okinawa, Japan); URM-P 39141, male, 405 mm TL (Okinawa, Japan); URM-P 39142, male, 415 mm TL (Okinawa, Japan).

APPENDIX 2. GenBank accession numbers.

\begin{tabular}{|c|c|c|c|}
\hline \multirow{2}{*}{ deposit number } & \multicolumn{3}{|c|}{ GenBank accession number } \\
\hline & $16 \mathrm{~S}$ & COI & Cytb \\
\hline MSM-19-292 & LC517195 & LC517222 & LC517249 \\
\hline MSM-19-293 & LC517196 & LC517223 & LC517250 \\
\hline MSM-19-295 & LC517197 & LC517224 & LC517251 \\
\hline MSM-19-296 & LC517198 & LC517225 & LC517252 \\
\hline MSM-19-297 & LC517199 & LC517226 & LC517253 \\
\hline MSM-19-298 & LC5 517200 & LC5 17227 & LC517254 \\
\hline MSM-19-299 & LC517201 & LC517228 & LC517255 \\
\hline MSM-19-300 & LC517202 & LC517229 & LC517256 \\
\hline MSM-19-301 & LC517203 & LC517230 & LC517257 \\
\hline MSM-19-305 & LC517181 & LC517208 & LC517235 \\
\hline MSM-19-320 & LC517182 & LC517209 & LC517236 \\
\hline NSMT-P 78557 & LC517183 & LC5 17210 & LC517237 \\
\hline NSMT-P 135960 & LC517206 & LC517233 & LC517260 \\
\hline NSMT-P 135961 & LC517193 & LC5 17220 & LC517247 \\
\hline NSMT-P 135962 & LC517194 & LC517221 & LC517248 \\
\hline NSMT-P 135963 & LC517192 & LC517219 & LC517246 \\
\hline NSMT-P 135964 & LC517190 & LC517217 & LC517244 \\
\hline NSMT-P 135965 & LC517207 & LC517234 & LC517261 \\
\hline NSMT-P 136479 & LC517184 & LC517211 & LC517238 \\
\hline NSMT-P 136480 & LC517185 & LC5 517212 & LC517239 \\
\hline SPMN-PI 45543 & LC517204 & LC517231 & LC517258 \\
\hline SPMN-PI 45544 & LC517205 & LC517232 & LC517259 \\
\hline SPMN-PI 45549 & LC517191 & LC517218 & LC517245 \\
\hline SPMN-PI 45568 & LC517186 & LC517213 & LC517240 \\
\hline SPMN-PI 45569 & LC517187 & LC517214 & LC517241 \\
\hline SPMN-PI 45570 & LC517188 & LC517215 & LC517242 \\
\hline SPMN-PI 45571 & LC517189 & LC517216 & LC517243 \\
\hline
\end{tabular}

